NBER WORKING PAPER SERIES

\title{
LAST-IN FIRST-OUT OLIGOPOLY DYNAMICS
}

\author{
Jaap H. Abbring \\ Jeffrey R. Campbell \\ Working Paper 14674 \\ http://www.nber.org/papers/w14674
NATIONAL BUREAU OF ECONOMIC RESEARCH
1050 Massachusetts Avenue
Cambridge, MA 02138
January 2009

We are grateful to Eugene Amromin, Gadi Barlevy, Allan Collard-Wexler, Meredith Crowley, and Richard Rosen, for their insightful comments; to Tom Holmes for his discussion at the 2006 Duke-Northwestern-Texas IO Theory Conference; and to R. Andrew Butters for superb research assistance. The National Science Foundation supported this research through Grant 0137042 to the NBER. De Jonge Akademie of the Royal Netherlands Academy of Arts and Sciences supported this research through a travel grant. The views expressed herein are those of the author(s) and do not necessarily reflect the views of the National Bureau of Economic Research.

NBER working papers are circulated for discussion and comment purposes. They have not been peerreviewed or been subject to the review by the NBER Board of Directors that accompanies official NBER publications.

(C) 2009 by Jaap H. Abbring and Jeffrey R. Campbell. All rights reserved. Short sections of text, not to exceed two paragraphs, may be quoted without explicit permission provided that full credit, including (C) notice, is given to the source. 
Last-In First-Out Oligopoly Dynamics

Jaap H. Abbring and Jeffrey R. Campbell

NBER Working Paper No. 14674

January 2009

JEL No. L13

\title{
ABSTRACT
}

This paper extends the static analysis of oligopoly structure into an infinite-horizon setting with sunk costs and demand uncertainty. The observation that exit rates decline with firm age motivates the assumption of last-in first-out dynamics: An entrant expects to produce no longer than any incumbent. This selects an essentially unique Markov-perfect equilibrium. With mild restrictions on the demand shocks, sequences of thresholds describe firms' equilibrium entry and survival decisions. Bresnahan and Reiss's (1993) empirical analysis of oligopolists' entry and exit assumes that such thresholds govern the evolution of the number of competitors. Our analysis provides an infinite-horizon game-theoretic foundation for that structure.

\author{
Jaap H. Abbring \\ CentER, Department of Econometrics \& OR \\ Tilburg University \\ P.O. Box 90153 \\ 5000 LE Tilburg, The Netherlands \\ and Tinbergen Institute \\ and Institute for the Study of Labor (IZA) \\ J.H.Abbring@uvt.nl \\ Jeffrey R. Campbell \\ Senior Economist \\ Economic Research Department \\ Federal Reserve Bank of Chicago \\ 230 South LaSalle Street \\ Chicago, IL 60604-1413 \\ and NBER \\ jcampbell@frbchi.org
}




\title{
Last-In First-Out Oligopoly Dynamics*
}

\author{
Jaap H. Abbring ${ }^{\dagger} \quad$ Jeffrey R. Campbell ${ }^{\ddagger}$
}

January, 2009

\begin{abstract}
This paper extends the static analysis of oligopoly structure into an infinite-horizon setting with sunk costs and demand uncertainty. The observation that exit rates decline with firm age motivates the assumption of last-in first-out dynamics: An entrant expects to produce no longer than any incumbent. This selects an essentially unique Markov-perfect equilibrium. With mild restrictions on the demand shocks, sequences of thresholds describe firms' equilibrium entry and survival decisions. Bresnahan and Reiss's (1993) empirical analysis of oligopolists' entry and exit assumes that such thresholds govern the evolution of the number of competitors. Our analysis provides an infinite-horizon game-theoretic foundation for that structure.
\end{abstract}

JEL Classification: L13.

Keywords: Sunk costs, demand uncertainty, Markov-perfect equilibrium, LIFO.

\section{Introduction}

This paper develops and presents a simple and tractable model of oligopoly dynamics based on the static entry game used by Bresnahan and Reiss (1990). A random number of consumers demands the industry's services, and this state evolves stochastically. Entry possibly requires paying a sunk cost, and continued operation incurs fixed costs. Incumbents who wish to avoid these per-period fixed costs in markets that are no longer profitable exit. Bresnahan and Reiss's (1993) empirical analysis of oligopolists' entry and exit assumes that thresholds govern the evolution of the number of competitors. That is, entry occurs whenever demand passes above one in a sequence of entry thresholds, and exit occurs if it subsequently passes below a corresponding exit threshold. A monopolist uses a threshold-based rule for entry and exit if raising current demand stochastically increases tomorrow's demand. We show that this condition alone does not guarantee that oligopolists use threshold rules, because a larger current market might make future entry more likely and consequently reduce an oligopolist's value. Nevertheless, we provide mild conditions on the demand process that guarantee that thresholds govern all firms' equilibrium entry and exit choices. In this way, our analysis provides an infinite-horizon game-theoretic foundation for Bresnahan and Reiss's (1993) empirical framework, which can be applied to extend their earlier structural estimation of static oligopoly models to a fully dynamic setting. The model makes a unique equilibrium prediction, which can be calculated very quickly. Thus, it can be used for policy experiments. This paper's companion (Abbring and Campbell, 2007) exemplifies this with an examination of how raising a barrier to entry for a second firm changes duopoly dynamics.

Bresnahan and Reiss (1991a) noted that the static oligopoly entry game can have multiple equilibria, which obviously complicates prediction. To select a unique equilibrium, both Bresnahan and Reiss (1990) and Berry (1992) assume that firms move sequentially. We take a similar approach by allowing older firms to

\footnotetext{
${ }^{*}$ We are grateful to Eugene Amromin, Gadi Barlevy, Allan Collard-Wexler, Meredith Crowley, and Richard Rosen, for their insightful comments; to Tom Holmes for his discussion at the 2006 Duke-Northwestern-Texas IO Theory Conference; and to R. Andrew Butters for superb research assistance. The National Science Foundation supported this research through Grant 0137042 to the NBER. De Jonge Akademie of the Royal Netherlands Academy of Arts and Sciences supported this research through a travel grant. A replication file is available at http://www.nber.org/ jrc/lifo.

${ }^{\dagger}$ CentER, Department of Econometrics \& OR, Tilburg University, P.O. Box 90153, 5000 LE Tilburg, The Netherlands; J.H.Abbring@uvt.nl

${ }^{\ddagger}$ Federal Reserve Bank of Chicago, 230 South LaSalle Street, Chicago, IL 60604, U.S.A., and NBER; jcampbell@frbchi.org.
} 
commit to continuation before their younger counterparts. We also restrict attention to equilibria in which firms correctly believe that no firm will produce after an older rival exits. That is, the equilibria have a last-in first-out (LIFO) structure. Three considerations motivate this focus. First, it is consistent with the widespread observation that young firms exit more frequently than their older counterparts. Second, the equilibrium approximates the "natural" Markov-perfect equilibrium in an extension of the model in which firms' costs decrease with age and the most efficient firms survive. Third and perhaps most importantly, this restriction vastly simplifies the equilibrium analysis. We prove that, irrespectively of whether the conditions for threshold rules are imposed or not, there always exists such an equilibrium and that it is (essentially) unique. This simplicity comes at one substantial cost: All shocks occur at the market level, so there is no reason for simultaneous entry and exit.

The model's theoretical simplicity makes it well-suited for exploring how parameter changes impact equilibrium dynamics and long-run market structure. To show this, we calculate the effects of increasing demand uncertainty on firms' equilibrium entry and exit thresholds. Nonstrategic analysis of the firm life cycle suggests that additional uncertainty should raise the value of the option to exit and thereby substantially lower both entry and exit thresholds. The oligopolistic exit thresholds do indeed fall with uncertainty, but the entry thresholds do not. Their relative invariance reflects an offsetting effect that a monopolist does not face: Increasing demand uncertainty raises the probability of further entry and thereby reduces a new firm's value. We also calculate the population "estimates" of oligopoly profit margins using the static ordered Probit procedure of Bresnahan and Reiss (1990) and data generated from the model's ergodic distribution. We find that the delay in exit arising from uncertainty (familiar from Dixit and Pindyck, 1994) biases these entry threshold estimates downwards, and this biases the estimated effect of additional competition on profits downward. That is, a long-run procedure that abstracts from relevant dynamic considerations can find "evidence" that profit margins decline with entry when in fact they are constant.

The sequential nature of firms' entry and exit decisions and the assumption that firms rationally expect LIFO dynamics substantially structures our analysis. In some previous work, the assumption that firms move sequentially commits early movers to their actions. Examples are Dixit's (1980) two-period Stackelberg investment game and Maskin and Tirole's (1988) infinite-horizon alternating-moves quantity game. In a finite-horizon game, ordering players' moves usually selects a unique subgame-perfect Nash equilibrium. Such sequencing need not select a single Markov-perfect equilibrium in an infinite-horizon setting like ours, so researchers sometimes structure expectations with assumptions - such as LIFO - to select a "natural" equilibrium. For example, Cabral (1993) assumes that younger firms that have not yet exploited the learning curve and therefore have high costs exit before their older low-cost counterparts. Modifying our model to make fixed costs decline deterministically with a firm's age is straightforward, and we obtain the LIFO equilibrium as a limit of the sequence of "natural" (in Cabral's sense) equilibria to our model as we send the learning curve's slope to zero. Thus, the LIFO equilibrium analysis seems useful for industries where incumbents enjoy small technological advantages over entrants.

Jovanovic (1982) and Hopenhayn (1992) provide analytic results for industry dynamics with many firms, but we know few similarly useful results for oligopolies. This apparent intractability has led researchers to approach questions of oligopoly dynamics computationally within Ericson and Pakes's (1995) framework for the empirical analysis of Markov-perfect oligopoly dynamics. Pakes and McGuire's (1994) algorithm for its equilibrium calculation iterates on a Bellman-like operator for the firms' value functions. It is well known that Bellman iteration converges slowly for conventional dynamic programming problems with high-dimensional states, so it should be no surprise that applying it to the oligopoly problem is computationally expensive.

The proofs of equilibrium existence and uniqueness for our model proceed constructively, beginning with the survival decision for the youngest firm when no further entry can be profitable even at the highest possible demand realization. Because no incumbents exit during its lifetime, this survival decision corresponds to a standard dynamic programming problem with only the current demand as a state variable. With this in hand, we can determine the entry choice for any firm that would occupy this "final" position. Given these entry and survival decisions, the survival problem for a firm expecting at most one more entrant corresponds to a dynamic programming problem with two state variables, current demand and the presence or absence of a younger competitor. Proceeding recursively in this way yields the unique Markov-perfect equilibrium decision rules and firm values with a LIFO structure. In this paper's companion (Abbring and Campbell, 2007), we have used this construction to analytically characterize the effects of raising late entrants' sunk costs and to calculate equilibria for hundreds of parameter values. Because the involved dynamic programming 
problems have a small state space, these calculations together take only a few minutes.

The remainder of this paper proceeds as follows. The next section presents the model's primitives and demonstrates the uniqueness of a Markov-perfect equilibrium with a LIFO structure. To clarify how the model's moving parts fit together, that section closes with an examination of a particular specification for the demand shocks that yields a pencil-and-paper solution. Section 3 gives sufficient conditions for firms to use threshold rules for their equilibrium entry and exit decisions, and Section 4 illustrates the model's application with the investigations of the dependence of oligopolists' entry and exit thresholds on demand uncertainty and of Bresnahan and Reiss's (1990) static estimation procedure.

\section{The Model}

The model consists of a single oligopolistic market in discrete time $t$. There is a countably infinite number of firms that are potentially active in the market. We index these firms by $j \in \mathbb{N}$, and below we refer to $j$ as the firm's name. At time $0, N_{0}=0$ firms are active. Entry and subsequent exit determine the number of active firms in each later period, $N_{t}$. The number of consumers in the market, $C_{t}$, evolves exogenously according to a first-order Markov process bounded between $\hat{C} \geq 0$ and $\check{C}<\infty$. We denote the conditional distribution of $C_{t}$ with $Q\left(c \mid C_{t-1}\right) \equiv \operatorname{Pr}\left[C_{t} \leq c \mid C_{t-1}\right]$.

Figure 1 illustrates the sequence of events and actions within a period using a portion of the game tree. It begins with the inherited values of $C_{t-1}$ and $N_{t}$ and with the name of the first potential entrant, $J_{t} .{ }^{1}$ All participants observe the realization of $C_{t}$, and all active firms receive profits equal to $\left(C_{t} / N_{t}\right) \times \pi\left(N_{t}\right)-\kappa$. Here, each firm serves $C_{t} / N_{t}$ consumers, and $\pi\left(N_{t}\right)$ is the producer surplus earned from each one. ${ }^{2}$ Increasing $N_{t}$ weakly decreases $\pi\left(N_{t}\right)$. The term $\kappa>0$ represents fixed costs of production.

After serving the market, active firms decide whether they will remain so. Exit is irreversible but otherwise costless. It allows the firm to avoid future periods' fixed production costs. If firm $j$ is active in this period, denote the rank of its name in the set of all active firms' names with $R_{t}^{j}$. This equals one for the firm with the lowest name, and it equals $N_{t}$ for the firm with the highest name. The active firms' continuation decisions proceed sequentially in increasing order of this rank. Firms can use mixed strategies, so we describe their choices with the survival probability $a$. These strategies' pure realizations also occur sequentially, so a firm later in the sequence conditions on its rivals' realized (binary) continuation choices.

After active firms' continuation decisions, those firms that have not yet had an opportunity to enter make entry decisions in the order of their names, starting with $J_{t}$. These continue until one potential entrant remains out of the industry. The first potential entrant for the next period, $J_{t+1}$, has this firm's name plus one. Because entry decisions proceed sequentially in increasing order of the firms' names, any entrant $j$ will have a rank $R_{t+1}^{j}$ greater than that of any surviving incumbent. Its cost of entry is $\varphi\left(R_{t+1}^{j}\right)$. We assume that $\varphi\left(R_{t+1}^{j}\right) \geq 0$ and is weakly increasing in $R_{t+1}^{j}$. This allows for, but does not require, later entrants to face a "barrier to entry" in the form of elevated sunk costs. ${ }^{3}$ The payoff to staying out of the industry is always zero, because a firm with an entry opportunity cannot delay its choice. Both active firms' and potential entrants' decisions maximize their expected stream of profits discounted with a factor $\beta<1$.

Before proceeding, we wish to highlight how the environment gives older firms priority in committing to continuation. Entry decisions proceed sequentially in increasing order of the firms' names; so active firms' values of $R_{t}^{j}$ rank their ages as well as their names if they entered in distinct periods. In this case, the ordering of continuation decisions by $R_{t}^{j}$ puts the oldest firm first and the youngest firm last. We employ the convention of assigning $R_{t}^{j}$ based on firms' names to break any ties that occur when two or more firms enter the market in the same period.

\subsection{Markov-Perfect Equilibrium}

We choose as our equilibrium concept symmetric Markov-perfect equilibrium. When firm $j$ decides whether to stay or exit, $N_{t}-R_{t}^{j}$ (the number of active firms following it in the sequence), $C_{t}$, and $R_{t+1}^{j}$ (its rank

\footnotetext{
${ }^{1}$ We initialize this with $J_{0}=0$.

${ }^{2}$ This section's analysis extends directly to a model in which a vector-valued Markov process $C_{t}$ influences total producer surplus in a general way. In particular, if we replace $C_{t} \pi\left(N_{t}\right)$ for scalar $C_{t}$ with $\pi\left(C_{t}, N_{t}\right)$ for vector-valued $C_{t}$, this section's equilibrium existence and uniqueness results continue to hold without change.

${ }^{3}$ This feature of the model is the focus of the companion paper, Abbring and Campbell (2007).
} 


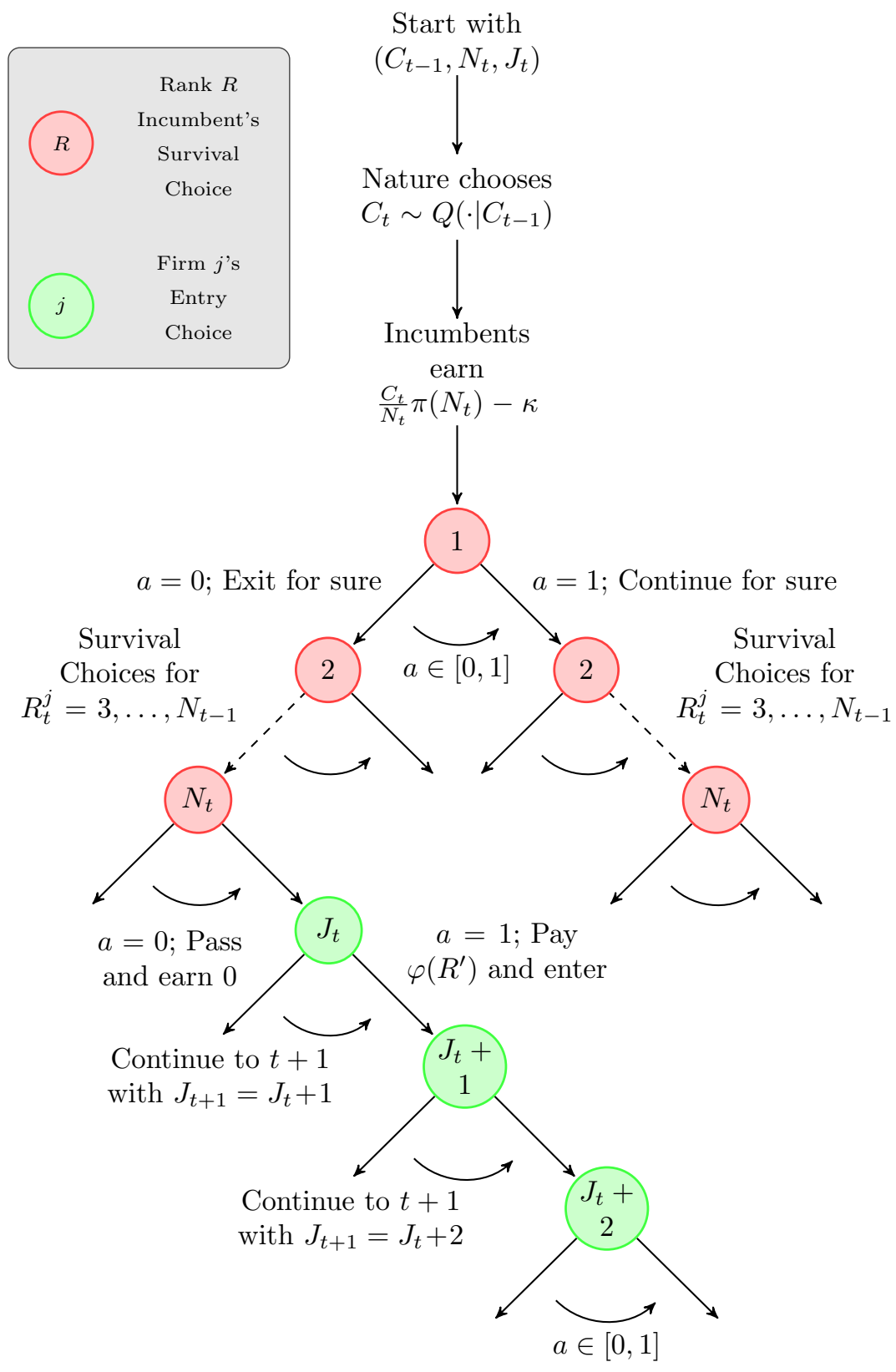

Figure 1: The game tree within a period.

All firms have names $j \in \mathbb{N}$. The rank $R_{t}^{j}$ orders active firms in increasing order of their names, and $J_{t}$ gives the name of the first firm with an entry opportunity in period $t$. All firms discount future payoffs with $\beta<1$. All firms use mixed strategies (prescribing actions denoted with $a \in[0,1]$ ), and the tree's branches follow their pure outcomes. See the text for further details. 
in the next period's sequence of active firms) are available and payoff-relevant. Collect these into $H_{j t} \equiv$ $\left(N_{t}-R_{t}^{j}, C_{t}, R_{t+1}^{j}\right)$. Similarly, the payoff-relevant state to a potential entrant is $H_{j t} \equiv\left(C_{t}, R_{t+1}^{j}\right)$. Note that $H_{j t}$ takes its values in $\mathcal{H}_{S} \equiv \mathbb{Z}_{+} \times[\hat{C}, \check{C}] \times \mathbb{N}$ for firms active in period $t$ and in $\mathcal{H}_{E} \equiv[\hat{C}, \check{C}] \times \mathbb{N}$ for potential entrants. Here and below, we use $S$ and $E$ to denote potential survivors and entrants.

A Markov strategy for firm $j$ is a pair of functions $A_{S}^{j}: \mathcal{H}_{S} \rightarrow[0,1]$ and $A_{E}^{j}: \mathcal{H}_{E} \rightarrow[0,1]$. These represent the probabilities of being active in the next period given that the firm is currently active $\left(A_{S}^{j}\right)$ and given that the firm has an entry opportunity $\left(A_{E}^{j}\right)$. We say that $A_{S}^{j}$ and $A_{E}^{j}$ are pure if they are $\{0,1\}$-valued. A symmetric Markov-perfect equilibrium is a subgame-perfect equilibrium in which all firms follow the same Markov strategy.

When firms use Markov strategies, the payoff-relevant state variables determine an active firm's expected discounted profits, which we denote with $v\left(H_{S}\right)$. In a Markov-perfect equilibrium, these satisfy the Bellman equation

$$
v\left(H_{S}\right)=\max _{a \in[0,1]} a \beta \mathbb{E}\left[\frac{C^{\prime}}{N^{\prime}} \pi\left(N^{\prime}\right)-\kappa+v\left(H_{S}^{\prime}\right) \mid H_{S}\right] .
$$

Here and throughout, we adopt conventional notation and denote the variable corresponding to $X$ in the next period with $X^{\prime}$. In Equation (1), the expectation of $N^{\prime}$ is calculated using all firms' strategies conditional on the particular firm of interest choosing to be active.

It is well known that multiple Markov-perfect equilibria can exist in similar models. ${ }^{4}$ To overcome this standard difficulty, we restrict attention to equilibria in which firms' entry and exit policies arise from a last-in first-out (LIFO) strategy.

Definition 1. A LIFO strategy is a strategy $\left(A_{S}, A_{E}\right)$ such that $A_{S}$ is pure, with $A_{S}\left(N-R, C, R^{\prime}\right)$ weakly decreasing in $R$.

If all firms adopt a common LIFO strategy $\left(A_{S}, A_{E}\right)$, then an active firm with rank $R \geq 2$ never stays if the predecessor in the sequence of active firms exits, because

$$
A_{S}\left(N-R, C, R^{\prime}\right)=0 \Longrightarrow A_{S}\left(N-R-1, C, R^{\prime}\right)=0 .
$$

As a consequence, if firms adopt a common LIFO strategy, they exit in the reverse order of their entry. Conversely, if firms use a common strategy and always exit in the reverse order of their entry, then the common strategy is a LIFO strategy.

Empirical studies of industry dynamics consistently find that young firms exit more frequently than their older rivals. For example, Dunne, Roberts, and Samuelson (1988) examined the survival of firms that entered manufacturing between the 1963 and 1967 Economic Censuses and lived to be sampled in 1967. Sixty four percent of these exited between 1967 and 1972. Their exit rate for the next five years was still substantial but considerably lower, 42 percent. This cohort's exit rate for the five years after that equalled 40 percent. Jarmin, Klimek, and Miranda (2003) calculated analogous five-year exit rates for firms that entered retail trade between 1977 and 1982 and lived until 1982. Of these, 60 percent exited by 1987. Their exit rates for 1987-1992 and 1992-1997 were 50 percent and 40 percent, so the decline of exit rates occurred more gradually for that cohort. The restriction to LIFO strategies mimics these declining exit rates in an extreme way, because the youngest firm always exits first. We do not doubt that firms sometimes outlast their older competitors, but the pervasive finding that exit rates decline with an entry cohort's age leads us to believe that the restriction to LIFO strategies provides a useful point of departure for examining dynamic oligopolies.

The payoff from restricting attention to LIFO strategies begins with the following proposition.

Proposition 1. There exists a symmetric Markov-perfect equilibrium in a LIFO strategy $\left(A_{S}, A_{E}\right)$ such that $A_{S}\left(N-R, C, R^{\prime}\right)$ is pure, constant in $N-R$, and weakly decreasing in $R^{\prime} ; A_{E}\left(C, R^{\prime}\right)$ is pure and weakly decreasing in $R^{\prime}$; and

$$
A_{S}\left(N-R, C, R^{\prime}\right) \geq A_{E}\left(C, R^{\prime}\right)
$$

\footnotetext{
${ }^{4}$ See Doraszelski and Satterthwaite (2007).
} 
The equilibrium survival and entry probabilities in Proposition 1 weakly decrease with the firm's rank in the next period. Moreover, the survival probability is constant in the number of firms with unresolved continuation decisions, and weakly larger than the entry probability of a firm with the same prospective rank in the same demand state.

This paper's appendix contains the proposition's constructive proof, which has two critical steps. First, we note that the upper bound on $C$ implies that the number of firms that ever produce in a Markov-perfect equilibrium cannot exceed some bound, which we call $\check{N}$. Because a firm with rank $\check{N}$ expects none of its older competitors to cease production before it does, this firm's optimal exit rule corresponds to that from a simple dynamic programming problem. Second, we solve exit decision problems for firms with ranks $\check{N}-1, \check{N}-2, \ldots, 1$ that embody the assumption that other firms follow a LIFO strategy. A firm with rank $R$ forms its expectations about the behavior of firms with higher ranks using the solutions of those firms' decision problems. With the solutions to these standard dynamic programming problems in hand, we construct a candidate LIFO strategy and then verify that it satisfies the proposition's conditions and forms a Markov-perfect equilibrium. ${ }^{5}$

The existence proof strongly suggests that the Markov-perfect equilibrium in a LIFO strategy is unique, because the decision problems used in its construction have Bellman equations with unique solutions. However, we might be able to construct multiple LIFO equilibria by varying a firm's actions in states of indifference between activity and inactivity. We sidestep this difficulty by concentrating on equilibria in which a firm defaults to inactivity.

Definition 2. A symmetric Markov-perfect equilibrium strategy $\left(A_{S}, A_{E}\right)$ defaults to inactivity if $A_{S}\left(H_{S}\right)=$ 0 whenever $v\left(H_{S}\right)=0$ and $A_{E}\left(C, R^{\prime}\right)=0$ whenever $v\left(0, C, R^{\prime}\right)=\varphi\left(R^{\prime}\right)$.

Proposition 2. There exists a unique symmetric Markov-perfect equilibrium in a LIFO strategy that defaults to inactivity. This equilibrium's survival rule $A_{S}$ and entry rule $A_{E}$ are such that $A_{S}\left(N-R, C, R^{\prime}\right)$ is pure, constant in $N-R$, and weakly decreasing in $R^{\prime} ; A_{E}\left(C, R^{\prime}\right)$ is pure and weakly decreasing in $R^{\prime}$; and $A_{S}\left(N-R, C, R^{\prime}\right) \geq A_{E}\left(C, R^{\prime}\right)$.

Note that the proposition's requirement that the equilibrium strategy defaults to inactivity ensures that it is pure. ${ }^{6}$

Other symmetric Markov-perfect equilibria that default to inactivity might exist, but in them the apparent advantage of early entrants to commit to continuation does not always translate into longevity. Henceforth, we constrain our attention to the unique symmetric Markov-perfect equilibrium in a LIFO strategy that defaults to inactivity. ${ }^{7}$

\subsection{A Pencil-and-Paper Example}

If we assume that $C_{t}=C_{t-1}$ with probability $1-\lambda$ and that it equals a draw from a uniform distribution on $[\hat{C}, \check{C}]$ with the complementary probability, then we can calculate the model's equilibrium value functions and decision rules with pencil and paper. Before proceeding, we examine this special case to illustrate the model's moving parts. For further simplification, suppose that $\pi(N)=0$ for $N \geq 3$, so at most two firms serve the industry. To ensure that the equilibrium dynamics are not trivial, we also assume that no firm

\footnotetext{
${ }^{5}$ Amir and Lambson (2003) prove existence of a subgame-perfect equilibrium in an infinite-horizon model that is similar to ours, but in which firms move simultaneously in each stage game. They do so by constructing an equilibrium that is the limit of a sequence of LIFO equilibria in the finite-horizon versions of their model as the horizon grows to infinity. This suggests an alternative interpretation of our LIFO equilibrium as the limit of the sequence of equilibria from our model's finite-horizon analogues.

${ }^{6}$ Relaxing the assumption that $\varphi$ is weakly increasing might be of some interest. After removing the conclusion that $A_{E}\left(C, R^{\prime}\right)$ weakly decreases in $R^{\prime}$ from Propositions 1 and 2 , both propositions continue to hold good when $\varphi\left(R^{\prime}\right)$ may decrease with $R^{\prime}$.

${ }^{7}$ In the model, $\pi(N)$ and $\kappa$ depend neither on the firm's name nor on its history. The previous literature on industry dynamics suggests three ways of relaxing this. The profitability of firms could be heterogeneous ex ante, drawn from some distribution upon entry, or improve with experience in the market. We can extend the model in these directions if the modifications never make a younger firm more profitable than any older rival. For example, we can incorporate irreproducible firm-specific capabilities by assigning higher fixed costs to firms with higher names. Drawing, upon entry, a firm's fixed cost from a twopoint distribution with a very large higher value would also leave the model's LIFO structure intact. Such shocks can create simultaneous entry and exit as potential entrants sequentially try to draw a low fixed cost. The learning curve mentioned in this paper's introduction exemplifies firm-specific changes in profits after entry.
} 
will serve the industry if demand is low enough and that two firms will serve the industry if it is sufficiently high. ${ }^{8}$

To begin, consider an incumbent firm with rank 2. In an equilibrium in a LIFO strategy, its profit equals $(C / 2) \pi(2)-\kappa$. It will earn this until the next time that $C_{t}$ changes, at which point the new demand value will be statistically independent of its current value. It is straightforward to use these facts to show that this firm's value function is the following piecewise linear function of $C$ :

$$
v(0, C, 2)= \begin{cases}0 & \text { if } C \leq \underline{C}_{2}, \\ \beta \frac{(1-\lambda)\left(\frac{C}{2} \pi(2)-\kappa\right)+\lambda \tilde{v}(0,2)}{1-\beta(1-\lambda)} & \text { if } C>\underline{C}_{2},\end{cases}
$$

where

$$
\widetilde{v}(0,2)=\frac{1}{2}\left(\frac{\hat{C}+\check{C}}{2}\right) \pi(2)-\kappa+\int_{\hat{C}}^{\check{C}} \frac{v\left(0, C^{\prime}, 2\right)}{(\check{C}-\hat{C})} d C^{\prime} .
$$

Here, $\widetilde{v}(0,2)$ is the firm's expected value following a new draw of $C_{t}$ and $\underline{C}_{2}$ is the largest value of $C$ that satisfies $v(0, C, 2)=0$. Optimality requires the firm to exit if $C<\underline{C}_{2}$. This value function is monotonic in $C$, so there is a unique entry threshold $\bar{C}_{2}$ that equates the continuation value with the entry cost. Thus, a second duopolist enters whenever $C_{t}$ exceeds $\bar{C}_{2}$ and exits if it subsequently falls at or below $\underline{C}_{2}$.

Next, consider the problem of an incumbent with rank 1. If this firm is currently a monopolist, it expects to remain so until $C_{t}>\bar{C}_{2}$; and if it is currently a duopolist, it expects to become a monopolist when $C_{t}$ falls below $\underline{C}_{2}$. This firm's value function is also piecewise linear. If the firm begins the period as the sole incumbent, it is

$$
v(0, C, 1)= \begin{cases}0 & \text { if } C \leq C_{1}, \\ \beta \frac{(1-\lambda)(C \pi(1)-\kappa)+\lambda \widetilde{v}(0,1)}{1-\beta(1-\lambda)} & \text { if } \underline{C}_{1}<C \leq \bar{C}_{2}, \\ \beta \frac{(1-\lambda)\left(\frac{C}{2} \pi(2)-\kappa\right)+\lambda \widetilde{v}(1,1)}{1-\beta(1-\lambda)} & \text { if } C>\bar{C}_{2} ;\end{cases}
$$

and if it begins as one of two incumbents it equals

$$
v(1, C, 1)= \begin{cases}0 & \text { if } C \leq \underline{C}_{1}, \\ \beta \frac{(1-\lambda)(C \pi(1)-\kappa)+\lambda \widetilde{v}(0,1)}{1-\beta(1-\lambda)} & \text { if } \underline{C}_{1}<C \leq \underline{C}_{2}, \\ \beta \frac{(1-\lambda)\left(\frac{C}{2} \pi(2)-\kappa\right)+\lambda \widetilde{v}(1,1)}{1-\beta(1-\lambda)} & \text { if } C>\underline{C}_{2} .\end{cases}
$$

The exit threshold $\underline{C}_{1}$ is the greatest value of $C$ such that $v(0, C, 1)=0$, and the expected values following a change in $C_{t}$ for a monopolist and a duopolist are

$$
\begin{aligned}
\widetilde{v}(0,1) & =\left(\frac{\hat{C}+\check{C}}{2}\right) \pi(1)-\kappa+\int_{\hat{C}}^{\check{C}} \frac{v\left(0, C^{\prime}, 1\right)}{(\check{C}-\hat{C})} d C^{\prime}, \\
\widetilde{v}(1,1) & =\frac{1}{2}\left(\frac{\hat{C}+\check{C}}{2}\right) \pi(2)-\kappa+\int_{\hat{C}} \frac{v\left(1, C^{\prime}, 1\right)}{(\check{C}-\hat{C})} d C^{\prime} .
\end{aligned}
$$

The value function of a firm with rank 1 does not always increase with $C$, because slightly raising $C$ from $\bar{C}_{2}$ induces entry by the second firm and causes both current profits and the continuation value to discretely drop. Nevertheless we know that they drop to a value above $\varphi(1)$, because at this point the second firm chooses to enter. Hence, it is still possible to find a unique entry threshold $\bar{C}_{1}$ that equates the value of entering with rank 1 to the cost of doing so.

\footnotetext{
${ }^{8}$ Sufficient conditions for these two properties are
}

$$
(1-\lambda)[\hat{C} \pi(1)-\kappa]+\lambda \frac{\frac{\hat{C}+\check{C}}{2} \pi(1)-\kappa}{1-\beta}<0 \quad \text { and } \quad \beta\left[\frac{(1-\lambda)\left(\frac{\check{C}}{2} \pi(2)-\kappa\right)+\frac{\lambda}{1-\beta}\left(\frac{1}{2} \frac{\check{C}+\hat{C}}{2} \pi(2)-\kappa\right)}{1-\beta(1-\lambda)}\right]>\varphi(2) .
$$


First Entrant's Value Function

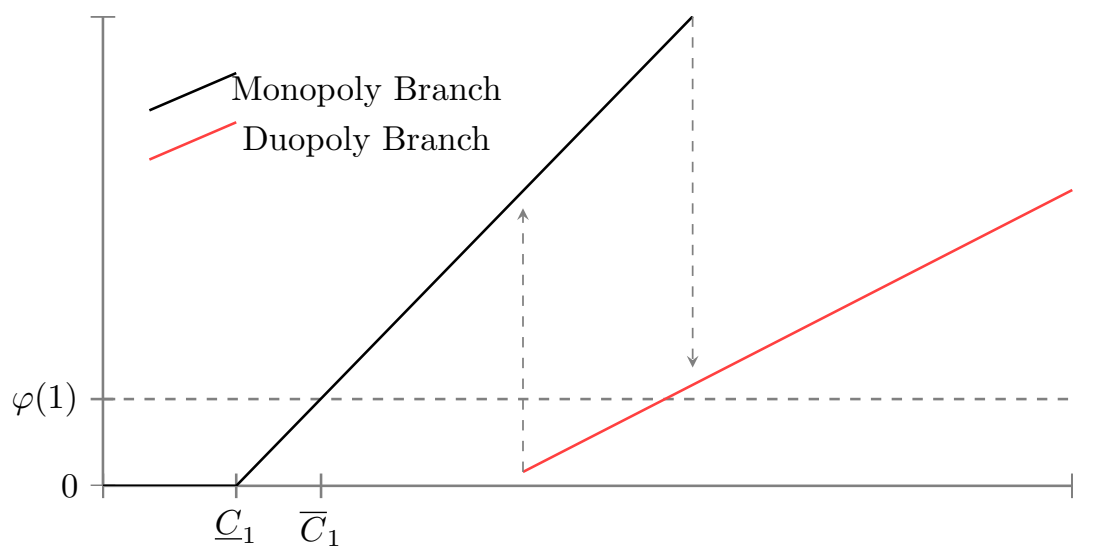

Second Entrant's Value Function

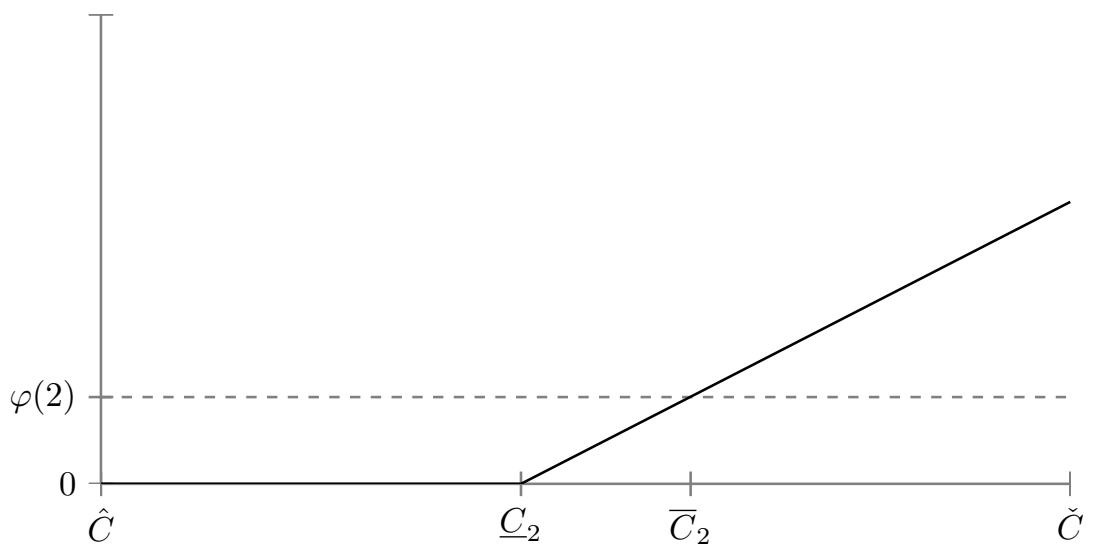

Figure 2: Equilibrium in the pencil-and-paper example.

In this example, $C_{t+1}=C_{t}$ with probability $1-\lambda$ and equals a draw from a uniform distribution over $[\hat{C}, \check{C}]$ with the complementary probability. A firm with the opportunity to become the second entrant takes it if $C>\bar{C}_{2}$, and that firm subsequently exits if $C \leq \underline{C}_{2}$. The value of the first firm to enter is discontinuous. As a function of $C_{t}$, the value of such a firm starting the period without a competitor begins at the origin, proceeds horizontally to $\underline{C}_{1}$, and then follows the monopoly branch until $\bar{C}_{2}$. It then discontinuously drops to the duopoly branch and follows it until $C$ reaches $\check{C}$. The first entrant's value when facing an active competitor follows the same path except that the discontinuous fall occurs at $\underline{C}_{2}$ instead of $\bar{C}_{2}$. The grey arrows indicate the fall in the firm's value upon entry of a second firm and the increase in value when that competitor exits. Please see the text for further details. 
Figure 2 visually represents the equilibrium. In each panel, $C$ runs along the horizontal axis. The vertical axis gives the value of a firm when entry and exit decisions are made. The top panel plots the value of a firm with rank 1, while the bottom panel plots the value for a competitor with rank 2 . The value of a duopolist with rank 2 equals zero for $C<\underline{C}_{2}$, and thereafter increases linearly with $C$. The entry threshold $\bar{C}_{2}$ equates this value with $\varphi(2)$. The value of a firm with rank 1 has two branches. The monopoly branch gives the value of a monopolist when $C \leq \bar{C}_{2}$. If $C$ increases above $\bar{C}_{2}$ and thus induces entry, the firm's value drops to the duopoly branch. This has the same slope as the value function in the lower panel. Its intercept is higher, because the incumbent expects to eventually become a monopolist the first time that $C$ passes below $\underline{C}_{2}$. When this occurs, the firm's value returns to the monopoly branch. The entry and exit thresholds for this firm occur where the monopoly branch intersects $\varphi(1)$ and 0 .

The paper-and-pencil example provides a useful basic framework for analytically characterizing the effects of policy interventions in a dynamic duopoly. This paper's companion (Abbring and Campbell, 2007) uses this to determine the effects of raising a barrier to entry in a monopoly by increasing a second entrant's sunk costs, and to explore the consequences of replacing the LIFO assumption with a first-in first-out (FIFO) assumption.

\section{Threshold Entry and Exit Rules}

In the paper-and-pencil example, all firms follow threshold rules for their entry and continuation decisions.

Definition 3. A firm with rank $R^{\prime}$ follows a threshold rule if there exist real numbers $\underline{C}_{R^{\prime}}$ and $\bar{C}_{R^{\prime}}$ such that

$$
A_{S}\left(N-R, C, R^{\prime}\right)=I\left\{C>\underline{C}_{R^{\prime}}\right\} \quad \text { and } \quad A_{E}\left(C, R^{\prime}\right)=I\left\{C>\bar{C}_{R^{\prime}}\right\} .
$$

With such a rule, a potential entrant into a market with $R^{\prime}-1$ incumbents actually enters if and only if $C>\bar{C}_{R^{\prime}}$, and this firm exits the first time thereafter that $C \leq \underline{C}_{R^{\prime}}$.

There are three reasons to care about whether or not firms follow threshold rules. First, they pervade theoretical and empirical industrial organization. Second, they simplify the model's analysis, as the penciland-paper example illustrated. Third, as the next proposition shows, higher realizations of demand always result in more active firms in our model if and only if all firms use threshold rules. A monotonic influence of $C_{t}$ on $N_{t+i}, i>0$, appeals to us as "natural". 9

Proposition 3. Consider an initial condition $\left(N_{t}, C_{t}\right)$; a sequence of subsequent demand realizations $C_{t+1}, \ldots, C_{t+i-1}$; and the corresponding numbers of operating firms from the equilibrium of Proposition 2; $N_{t+1}, \ldots, N_{t+i}$. Increasing $C_{t}$ weakly increases $N_{t+i}$ for positive $i$ and any possible initial condition and sequence of subsequent demand realizations if and only if firms of all ranks follow threshold rules.

Appendix B presents the proposition's proof.

With stochastic monotonicity- $Q(\cdot \mid C)$ weakly decreases with $C$ - the value function for the firm with the highest possible rank weakly increases with $C$. Therefore, such a firm must follow a threshold rule. ${ }^{10}$ Hopenhayn (1992) uses this to demonstrate the existence of an optimal exit threshold for competitive firms. The Poisson innovation process from the pencil-and-paper example displays stochastic monotonicity, but the lower ranked firm's value function falls when a second competitor enters. Although that firm's value function is not monotonic, thresholds still govern its entry and exit. Thus, one might hope that the firms in our model use threshold rules when $C$ displays stochastic monotonicity. We show that this is not the case with the following numerical example.

Let us set $\check{N}=2$ and place $\ln C_{t} \in[-1.50,1.50]$. Suppose that the probability of $\ln C_{t}$ remaining unchanged is $1 / 2$. With probability $1 / 4$ it falls to the maximum of $\ln C_{t}-0.30$ and $\ln \hat{C}$, and with the same

\footnotetext{
${ }^{9} \mathrm{~A}$ threshold rule is only well-defined if $C_{t}$ is a scalar, so this section's analysis does not apply to the generalization to vector-valued $C_{t}$ mentioned in Footnote 2.

${ }^{10}$ Later in this section, we will make an additional left-continuity assumption, Assumption 4 , that ensures that this rule has Definition 3's strict threshold-crossing representation if it defaults to inactivity.
} 
probability it rises to the minimum of $\ln C_{t}+0.30$ and $\ln \check{C}$. That is,

$$
Q(c \mid C)= \begin{cases}0 & \text { if } \ln c<\max \{\ln C-0.30,-1.50\}, \\ 1 / 4 & \text { if } \max \{\ln C-0.30,-1.50\} \leq \ln c<\ln C, \\ 3 / 4 & \text { if } \ln C \leq \ln c<\min \{\ln C+0.30,1.50\}, \text { and } \\ 1 & \text { otherwise. }\end{cases}
$$

The model's other parameters in this example are $\varphi(1)=\varphi(2)=10, \pi(N)=2 \times I\{N \leq 2\}, \kappa=1$, and $\beta=1.05^{-1}$. Figure 3 shows both firms value functions. ${ }^{11}$ Clearly, the equilibrium entry rule for a firm with prospective rank 1 does not obey a threshold rule. Such a firm will enter in the disconnected sets $\mathcal{A}$ and $\mathcal{B}$, where the firm's value exceeds the cost of entry.

This example fails to produce threshold rules because a continuous increase in $C$ leads to a discontinuous jump in the probability of future entry: Crossing the upper boundary of $\mathcal{A}$ raises the probability of entry next period from 0 to $1 / 4$. The first entrant's value drops discretely, because the continuous increase in current profitability does not offset the loss from this increase in expected competition. In contrast, the probability of future entry was constant by design in the paper-and-pencil example.

Comparing the two examples suggests that firms will use threshold rules if the stochastic process limits this negative effect of increasing $C_{t}$ on expected future profits. Here, we present sufficient conditions on $Q(\cdot \mid C)$ for this to be so.

Assumption 1 (Monotonicity). $\mathbb{E}\left[C^{\prime} \mid C\right]$ is weakly increasing in $C$.

Assumption 2 (Independence). The innovation error $U^{\prime} \equiv C^{\prime}-\mathbb{E}\left[C^{\prime} \mid C\right]$ is independent of $C$.

Assumption 3 (Concavity). The cumulative distribution function $\tilde{Q}$ of $U^{\prime}$ is concave on $[\underline{U}, \infty)$, where $\underline{U} \equiv \inf _{C}\left\{C-\mathbb{E}\left[C^{\prime} \mid C\right]\right\}$.

Assumption 4 (Continuity). $\mathbb{E}\left[C^{\prime} \mid C\right]$ is continuous from the left.

Assumptions 1 and 2 together imply stochastic monotonicity. In particular, Assumption 2 is equivalent to $Q(c \mid C)=\tilde{Q}\left(c-\mathbb{E}\left[C^{\prime} \mid C\right]\right)$ for all $c$. With Assumption 1, this ensures that $Q(\cdot \mid C)$ weakly decreases with $C$.

Assumptions 2 and 3 together require $Q(\cdot \mid C)$ to be concave on $[C, \infty)$, because $c-\mathbb{E}\left[C^{\prime} \mid C\right] \geq C-$ $\mathbb{E}\left[C^{\prime} \mid C\right] \geq \underline{U}$ if $c \geq C$. Equivalently, $Q(\cdot \mid C)$ must have a weakly decreasing Lebesgue density to the right of $C$. The three-point distribution used to create Figure 3 satisfies Assumptions 1 and 2, but violates Assumption 3.

Assumption 4 implies that the value function in the equilibrium that defaults to inactivity is continuous from the left in $C$. It carries little substance, because Assumption 1 already implies that $\mathbb{E}\left[C^{\prime} \mid C\right]$ has at most countably many discontinuities.

With these assumptions in place, we can state this section's central result.

Proposition 4. Let $\left(A_{S}, A_{E}\right)$ be the unique symmetric Markov-perfect equilibrium in a LIFO strategy that defaults to inactivity. If $Q(\cdot \mid C)$ satisfies Assumptions 1-4, then firms with all ranks follow threshold policies.

The proposition's proof is given in Appendix B. Assumptions 2 and 3 ensure that the conditional distribution of $C^{\prime}$ has no mass points or modes to the right of $C$. Thus, increasing $C$ cannot move a "substantial" probability mass over another firm's entry threshold and cause a "discontinuous" increase in the probability of future entry (as in our example of a nonmonotonic exit rule). Together with Assumption 1, this suffices to guarantee that, for each given rank, the equilibrium value function crosses the cost of entry only once and is weakly increasing to the left of that crossing. The corresponding equilibrium strategy prescribes that firms enter and survive if demand exceeds rank-dependent entry and exit thresholds. Assumption 4 only ensures that the equilibrium strategy that defaults to inactivity has Definition 3's strict threshold-crossing representation.

To shed further light on the content of Assumption 3, suppose that $U^{\prime}$ has a uniform distribution with support $[-\sigma / 2, \sigma / 2]$. Assumption 3 requires $\mathbb{E}\left[C^{\prime} \mid C\right]-\sigma / 2 \leq C$ for all $C$. That is, the minimum possible realization of $C^{\prime}-C$ cannot exceed zero. In this sense, Assumption 3 bounds the growth of demand.

\footnotetext{
${ }^{11}$ For the computation, we restricted $C_{t}$ to a grid of 3000 evenly spaced points and used the algorithm described in Section 4.1.
} 

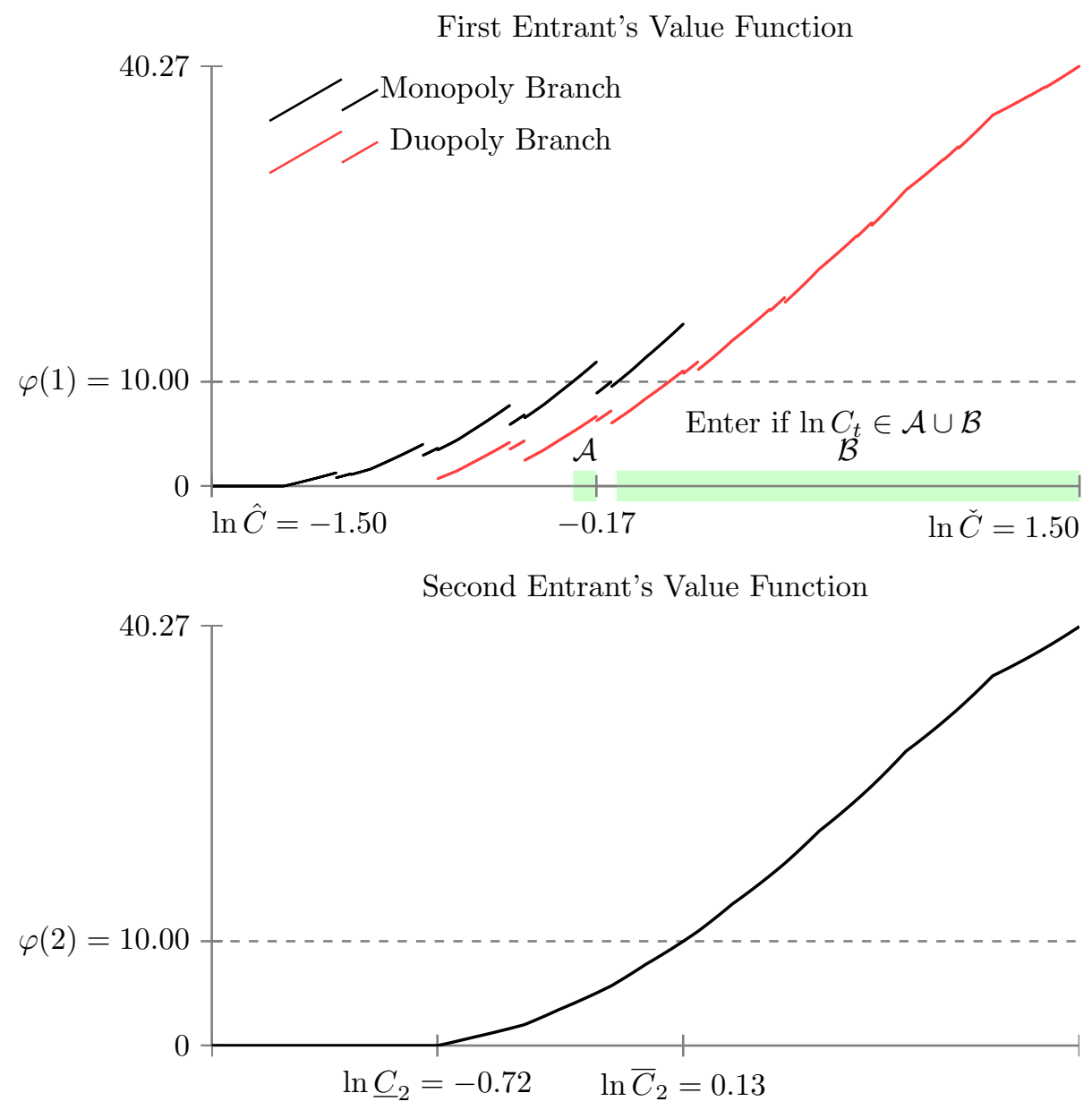

Figure 3: Example of LIFO equilibrium with a nonmonotonic entry rule.

In this example, $\ln C_{t+1}-\ln C_{t}$ equals zero with probability $1 / 2$ and equals either $\min \left\{0.30, \ln \check{C} / C_{t}\right\}$ or $\max \left\{-0.30, \ln C_{t} / \hat{C}\right\}$ with probability $1 / 4$ placed on each value. A firm with the opportunity to become the second entrant takes it if $\ln C_{t}>0.13$. The value of entry for a first entrant exceeds its cost if $\ln C_{t}$ is slightly less than $0.13-0.30=-0.17$. Increasing $\ln C_{t}$ from such a value pushes the highest possible realization of $\ln C_{t+1}$ past 0.13 . This discontinuously lowers the first entrant's value to a point below the cost of entry. Hence, the equilibrium entry strategy is not monotonic in $C_{t}$. Please see the text for further details. 
The Poisson innovation process from the pencil-and-paper example also satisfies the assumptions of Proposition 4. The following representation theorem demonstrates that these two examples are "typical" of such processes and aids in the construction of others.

Proposition 5. The transition function $Q(\cdot \mid C)$ satisfies Assumptions 1-3 if and only if there exist a sequence $Q^{1}(\cdot \mid C), Q^{2}(\cdot \mid C), \ldots$ of transition functions such that

$$
\lim _{K \rightarrow \infty} \sup _{c, C}\left|Q^{K}(c \mid C)-Q(c \mid C)\right|=0
$$

and; for all $K \in \mathbb{N}$;

$$
Q^{K}(\cdot \mid C)=\sum_{k=1}^{K} p_{k}^{K} Q_{k}^{K}(\cdot \mid C), \quad p_{1}^{K}, \ldots, p_{K}^{K} \geq 0, \quad \sum_{k=1}^{K} p_{k}^{K}=1 ;
$$

where; for $k=1, \ldots, K$;

$$
Q_{k}^{K}(c \mid C)= \begin{cases}0 & \text { if }\left(c-\mu_{k}^{K}(C)\right) / \sigma_{k}^{K} \leq-1 / 2 \\ \left(c-\mu_{k}^{K}(C)+\sigma_{k}^{K} / 2\right) / \sigma_{k}^{K} & \text { if }-1 / 2<\left(c-\mu_{k}^{K}(C)\right) / \sigma_{k}^{K} \leq 1 / 2 \\ 1 & \text { otherwise }\end{cases}
$$

and

$$
\begin{aligned}
& -\sigma_{k}^{K} \geq 0, \\
& -\mu_{k}^{K}(C) \text { weakly increases in } C, \\
& -\hat{C}+\sigma_{k}^{K} / 2 \leq \mu_{k}^{K}(C) \leq \check{C}-\sigma_{k}^{K} / 2 \text {, and } \\
& -\mu_{k}^{K}(C) \leq C+\sigma_{k}^{K} / 2 .
\end{aligned}
$$

This proposition's proof, in Appendix B, relies on the well-known fact that concave distributions can be represented by mixtures of uniform distributions with common lower ends of their supports.

Proposition 5 implies that each demand process that satisfies Assumptions 1-3 can be constructed by mixing uniform autoregressions that each satisfy monotonicity (as in Assumption 1) and the support condition used in the example. In particular, each of the mixed processes is a possibly nonlinear autoregression with conditional mean $\mu_{k}^{K}(C)$ and uniform innovations with standard deviation $\sigma_{k}^{K} / \sqrt{12}$. The coefficients $p_{k}^{K}$ give the mixing probabilities. The stochastic process from the pencil-and-paper example is such a mixture, with $\alpha$ and $1-\alpha$ serving as the mixing probabilities. In this case, one of the uniform distributions is degenerate at $C$. The support condition that $\mu_{k}^{K}(C) \leq C+\sigma_{k}^{K} / 2$ ensures that the current state is always in or above the support of each mixing distribution. With uniformity, this guarantees that $Q(\cdot \mid C)$ has a nonincreasing Lebesgue density on $(C, \check{C}]$ (Assumption 3).

To construct another example process, consider a random walk reflected at $\hat{C}$ and $\check{C}$. That is; set

$$
\mu_{k}^{K}(C)=\left\{\begin{array}{lll}
\hat{C}+\sigma_{k}^{K} / 2 & \text { if } \quad C \leq \hat{C}+\sigma_{k}^{K} / 2, \\
C & \text { if } \quad \hat{C}+\sigma_{k}^{K} / 2<C \leq \check{C}-\sigma_{k}^{K} / 2, \text { and } \\
\check{C}-\sigma_{k}^{K} / 2 & \text { if } \quad C>\check{C}-\sigma_{k}^{K} / 2
\end{array}\right.
$$

for some $0<\sigma_{k}^{K}<\check{C}-\hat{C}$. By mixing over such reflected random walks, we can approximate any symmetric and continuous distribution for the growth rate of demand in the region away from the boundaries of $[\hat{C}, \check{C}]$.

Proposition 5 does not cover Assumption 4. This assumption can directly be imposed by choosing $\mu_{k}^{K}(C)$ such that $\lim _{K \rightarrow \infty} \sum_{k=1}^{K} p_{k}^{K} \mu_{k}^{K}(C)$ is continuous from the left. In both the pencil-and-paper and the reflected random walk examples, this limit is continuous, and Assumption 4 is satisfied. 


\section{$4 \quad$ Entry and Exit with Uncertainty}

This section applies our analysis to two related questions: How does adding uncertainty impact oligopolists' entry and exit thresholds? How do estimates of oligopolists' producer surplus per consumer based on static models of the "long-run" without uncertainty or without sunk costs differ from their actual values?

Dixit and Pindyck (1994) review a large literature that characterizes competitive firms' entry and exit decisions with sunk costs and uncertain profits. Such a firm's value equals its fundamental value, the expected discounted profits from perpetual operation, plus the value of an option to sell this stream of (potentially negative) profits at a strike price of zero. This literature's key insight is that uncertainty about future profits raises the value of this put option and thereby decreases the frequency of exit. Abbring and Campbell (2006) estimated that this option value accounted for the majority of firm value in a particular competitive service industry. Our model allows us to investigate how the insights from this well-studied decision-theoretic problem apply to oligopolistic dynamics.

Our analysis of the second question follows a large literature based on static free-entry models of oligopoly structure, exemplified by Bresnahan and Reiss (1990, 1991b) and Berry (1992). They determined empirically how changing market size influenced the number of competitors using observations from cross-sections of local retail (Bresnahan and Reiss) and airline (Berry) markets. The models they used to structure their analysis can be viewed as versions of ours in which either demand remains unchanged over time or firms incur no sunk costs. These papers point to current demand as the key determinant of the number of firms: A market will attain $N$ firms if $N$ entrants can recover their fixed costs, but $N+1$ entrants cannot. These authors emphasize that the observed relationship between $C$ and $N$ depends on the rate at which $\pi(N)$ decreases (which Sutton, 1991, labeled the toughness of competition) and the rate at which $\varphi(N)$ increases (which McAfee, Mialon, and Williams, 2004, define to be an economic barrier to entry). If both of these functions are constant, then the number of active firms is roughly proportional to demand, $\bar{C}_{i}=i \times \bar{C}_{1}$. However, if either $\pi(N)$ decreases or $\varphi(N)$ increases, then $N / C$ declines with $C$. In this sense, increasing the toughness of competition or imposing an economic barrier to entry increases concentration.

Our approach to answering these questions is computational. Accordingly, we begin this section with an explicit presentation of the algorithm for equilibrium calculation. We then show how demand uncertainty impacts equilibrium entry and exit thresholds for a particular model parameterization. The section concludes with a presentation of the entry thresholds and the producer surplus per consumer calculated from feeding data generated by our model's ergodic distribution through a static Probit model of long-run equilibrium like that of Bresnahan and Reiss (1990, 1991b).

\subsection{Equilibrium Computation}

The proof of Proposition 1 outlines a simple algorithm for computing the Markov-perfect equilibrium of interest:

- Given values for the model's primitives, we choose an evenly spaced grid of values for $C$ in the interval $[\hat{C}, \breve{C}]$ and a Markov chain over this grid to approximate $Q(\cdot \mid C)$.

- We set $\check{N}$ equal to the largest value of $N$ such that

$$
\frac{\check{C}}{N} \pi(N)-\kappa \geq 0 .
$$

- We consider the decision problem of a firm with rank $\check{N}$. This firm rationally expects no further entry and sets $N^{\prime}$ equal to $N_{\tilde{N}}^{\prime}(X, C) \equiv \check{N}$ in all states $(X, C)$. Here, $X$ is the number of active firms with unresolved continuation decisions: $X=0$ for a potential entrant and $X=N-R$ for an active firm deciding on continuation. Under this supposition, we can solve the firm's dynamic programming problem by beginning with a trial value for its value function $v(0, \cdot, \tilde{N})$ and iterating on the Bellman operator in Equation (1) for $N=R=R^{\prime}=\check{N}$. This gives the firm's expected discounted profits $v(0, C, \check{N})$ for all $C$ on the chosen grid. In practice, this takes very, very little computer time. From $v(0, \cdot, \check{N})$, we can calculate the sets of demand states $C$ in which the firm chooses to enter and survive. We refer to these as the entry and survival sets

$$
\mathcal{E}_{\check{N}} \equiv\{C \mid v(0, C, \check{N})>\varphi(\check{N})\} \quad \text { and } \quad \mathcal{S}_{\check{N}} \equiv\{C \mid v(0, C, \check{N})>0\} .
$$


- The rest of the computation proceeds recursively for $R^{\prime}=\check{N}-1, \ldots, 1$. Suppose that we have computed entry sets $\mathcal{E}_{R^{\prime}+1}, \ldots, \mathcal{E}_{\check{N}}$ and survival sets $\mathcal{S}_{R^{\prime}+1}, \ldots, \mathcal{S}_{\check{N}}$. A firm with (prospective) rank $R^{\prime}$ rationally expects that these sets govern younger firms' entry and survival decisions, and that no firm will enter with rank larger than $\tilde{N}$. That is,

$$
N_{R^{\prime}}^{\prime}(X, C) \equiv R^{\prime}+\sum_{i=1}^{\check{N}-R^{\prime}}\left[I\left\{i \leq X, C \in \mathcal{S}_{R^{\prime}+i}\right\}+I\left\{i>X, C \in \mathcal{E}_{R^{\prime}+i}\right\}\right]
$$

governs the evolution of the number of firms; $X=0,1, \ldots, \check{N}-R^{\prime}$. With this specification for $N^{\prime}$ in place, we can solve this firm's dynamic programming problem by iterating on the Bellman operator in Equation (1) for given $R^{\prime}$, starting with e.g. the value function for a firm with rank $R^{\prime}+1$. This produces the expected discounted profits $v\left(X, \cdot, R^{\prime}\right)$, and the entry and survival sets

$$
\mathcal{E}_{R^{\prime}} \equiv\left\{C \mid v\left(0, C, R^{\prime}\right)>\varphi\left(R^{\prime}\right)\right\} \quad \text { and } \quad \mathcal{S}_{R^{\prime}} \equiv\left\{C \mid v\left(0, C, R^{\prime}\right)>0\right\}
$$

for a firm with rank $R^{\prime}$.

With the equilibrium entry and survival sets for all $\check{N}$ possible ranks in place, calculating observable aspects of industry dynamics (such as the ergodic distribution of $N_{t}$ ) is straightforward. Matlab programs and documentation are available in this article's replication file.

\subsection{Equilibrium Entry and Exit Thresholds}

With this algorithm, we have calculated the equilibrium entry and exit thresholds for a particular specification of the model that satisfies the sufficient conditions for firms to use threshold-based entry and exit policies. We set one model period to equal one year and chose $\beta$ to replicate a $5 \%$ annual rate of interest. We set $\kappa=1.75$ and $\varphi\left(R^{\prime}\right)=0.25 \beta /(1-\beta)$, so the fixed costs of a continuing establishment equal seven times sunk costs' rental equivalent value. We also set $\pi(N)=4$ for all $N$. With these parameter values and no demand uncertainty, the entry thresholds are $8 / 7$ of the corresponding exit thresholds and the entry threshold for a second firm equals one. We set $\hat{C}=e^{-1.5}, \check{C}=e^{1.5}$, and $Q(\cdot \mid C)$ to equal a mixture over 51 reflected random walks in the logarithm of $C$ with uniformly distributed innovations. The mixture approximates a normally distributed innovation. We denote the standard deviation of the normal distribution we seek to approximate with $\sigma$. Proposition 4 can be easily extended to the case where Assumptions 1-4 apply to a monotonic transformation of $C_{t}$, so the logarithmic specification for demand has no direct theoretical consequences. We choose it because population and income measures typically require a logarithmic transformation to display homoskedasticity across time.

The first two panels of Table 1 report the equilibrium entry and exit thresholds for this specification for four values of $\sigma: 0,0.05,0.10$, and 0.15. Given the support of $C_{t}$, up to eight firms could populate the industry if $\sigma=0$. Because $C_{t}$ is reflected off of $\check{C}$, demand displays mean reversion. Thus, such high states of demand are somewhat temporary if $\sigma>0$, and the maximum number of firms observed in the ergodic distribution accordingly decreases with $\sigma$. The two panels' cells for those missing firms' entry and exit thresholds are blank. ${ }^{12}$

Consider first the impact of increasing $\sigma$ on the entry thresholds. At least one firm enters an empty market with no demand uncertainty if $C_{t}>0.50$. This threshold hardly changes as $\sigma$ increases. Likewise, the entry threshold for a second firm remains very close to 1.00 as $\sigma$ rises. The thresholds for higher-ranked entrants all rise with $\sigma$ with one exception (to be discussed further below). Apparently, increasing demand uncertainty makes entry into an oligopoly less likely for a given value of $C_{t}$. Demand uncertainty has exactly the opposite impact on the entry of a potential monopolist. For such a firm, increasing uncertainty increases the value of the put option associated with exit, thereby raising profitability and lowering the firm's entry threshold.

\footnotetext{
${ }^{12}$ One way of gauging how much uncertainty these experiments embody is to calculate their entry and exit rates. When $\sigma=0$, the probability of a firm randomly drawn from the ergodic distribution exiting equals zero. This probability equals 0.5 percent, 1.1 percent, and 1.7 percent for the other three values of $\sigma$. These are annual exit rates, because we set $\beta$ so that one period equals one year.
} 
This difference between oligopolists' and monopolists' entry decisions arises from the threat of potential entry. A monopolist captures all of the increased profit from a favorable demand shock. For an oligopolist, further entry chops this right tail off of the profit distribution and thereby reduces the firm's option value. This explanation squares with the single exception to the rule that increasing $\sigma$ increases the entry threshold. Increasing $\sigma$ from 0.10 to 0.15 simultaneously eliminates the possibility that a sixth firm enters and reduces $\bar{C}_{5}$ from 2.70 to $2.59 .^{13}$ The third panel of Table 1 further illustrates this effect. It reports the equilibrium entry thresholds for the case where $\pi(N)=4 \times I\{N<5\}$, so that no more than four firms will populate the market. The entry thresholds for the first, second, and third firms are nearly identical to their values in the first panel. However, the entry thresholds for the fourth firm (facing no further entry) decline with $\sigma$.

Next examine the exit thresholds in the table's second panel. Without demand uncertainty, these form a line out of the origin with a slope approximately equal to 0.44. As expected, raising $\sigma$ decreases all of the exit thresholds. This mimics the well-known effect of increased uncertainty on monopolists' exit decisions: Uncertainty raises the value of the firm's put option, and exit requires this option's exercise. For completeness, Table 1 reports the equilibrium exit thresholds when $\pi(N)=4 \times I\{N<5\}$. As expected, this change has almost no impact on the exit thresholds for firms with ranks less than four. For the fourth firm, eliminating the possibility of further entry makes survival more attractive and thereby lowers the exit threshold even further.

To summarize, adding uncertainty either leaves the equilibrium entry thresholds unchanged or raises them somewhat. This result embodies two offsetting effects: Increasing uncertainty alone would make entry more attractive, but the accompanying increase in the probability of future entry reduces expected future profits. Changing the probability of future entry has comparatively little impact on the equilibrium exit thresholds, so adding uncertainty substantially reduces them.

\subsection{Static Analysis of Market Size and Entry}

We now characterize how a static "long-run" analysis of market size and entry interprets data generated by our dynamic model. For this, we modify our model to obtain a framework observationally equivalent to that estimated by Bresnahan and Reiss $(1990,1991 b)$. We begin by eliminating all but trivial dynamic considerations with the assumption that $C_{t}=C_{t-1}$ always. All markets begin with zero competitors, so removing demand uncertainty from the model eliminates exit. It also removes any meaningful distinction between fixed and sunk costs, so we set the latter to zero. This version of the model is econometrically degenerate because $C_{t-1}$ determines $N_{t}$ nonstochastically. We follow Bresnahan and Reiss and solve this problem by adding a random component to firms' fixed costs. Specifically, the fixed costs of any firm serving the market are $e^{\varepsilon} \kappa$, where $\varepsilon$ is a normally distributed across markets with mean 0 and variance $\varsigma^{2}$.

Free entry requires that all active firms earn a positive profit and that an additional firm would earn a nonpositive profit. ${ }^{14}$ That is

$$
\frac{C}{N} \times \pi(N)>e^{\varepsilon} \kappa \quad \text { and } \quad \frac{C}{N+1} \times \pi(N+1) \leq e^{\varepsilon} \kappa .
$$

For each $N=1, \ldots, \Sigma \bar{N}$; define the deterministic entry threshold $C_{N}^{\star}$ to be the unique solution to $(C / N) \pi(N)-$ $\kappa=0$. Exactly $N$ firms will serve the industry if $\ln C>\ln C_{N}^{\star}+\varepsilon$ and $\ln C \leq \ln C_{N+1}^{\star}+\varepsilon$. The probability that this occurs is

$$
\Phi\left(\frac{\ln \left(C / C_{N}^{\star}\right)}{\varsigma}\right)-\Phi\left(\frac{\ln \left(C / C_{N+1}^{\star}\right)}{\varsigma}\right) .
$$

In this expression, we set $\ln C_{0}^{\star}=-\infty$ and $\ln C_{\tilde{N}+1}^{\star}=\infty .^{15}$

\footnotetext{
${ }^{13}$ Entry by an eighth firm does not occur when there is demand uncertainty, so this discussion begs the question of why increasing $\sigma$ from 0 to 0.05 raises $\bar{C}_{7}$ from 3.49 to 3.61 . This change reflects the mean reversion noted above. The same principle explains the rise of $\bar{C}_{6}$ from 3.13 to 3.21 when $\sigma$ goes from 0.05 to 0.10 .

${ }^{14}$ The free entry conditions equal those from a model without dynamic considerations, because we have set sunk costs to zero. If we had assumed instead that the per period fixed costs equal zero and that the sunk $\operatorname{costs}$ equal $e^{\varepsilon} \varphi$, then the free entry conditions would be identical with $\varphi(1-\beta) / \beta$ replacing $\kappa$ everywhere.

${ }^{15}$ Note that more than $\check{N}$ firms will enter in the static model if $\pi(\check{N}+1)>0$ and the realization of $\varepsilon$ is sufficiently small. However, we will apply the static model to data generated from the dynamic model, which never have more than $\breve{N}$ active firms. For the purpose of this application, we can therefore ignore the possibility entry beyond $\check{N}$ firms.
} 
Table 1: Equilibrium Entry and Exit Thresholds ${ }^{\mathrm{a}}$

\begin{tabular}{|c|c|c|c|c|c|c|c|c|}
\hline \multicolumn{9}{|c|}{$\pi(N)=4$} \\
\hline$\sigma$ & \multicolumn{7}{|c|}{ Entry Thresholds } & $\bar{C}_{8}$ \\
\hline 0.00 & 0.50 & 1.00 & 1.50 & 1.99 & 2.50 & 2.99 & 3.49 & 3.99 \\
\hline 0.05 & 0.48 & 0.99 & 1.51 & 2.04 & 2.57 & 3.13 & 3.61 & \\
\hline 0.10 & 0.46 & 1.00 & 1.56 & 2.14 & 2.70 & 3.21 & & \\
\hline 0.15 & 0.46 & 1.02 & 1.62 & 2.19 & 2.59 & & & \\
\hline \multicolumn{9}{|c|}{ Exit Thresholds } \\
\hline$\sigma$ & $\underline{C}_{1}$ & $\underline{C}_{2}$ & $\underline{C}_{3}$ & $\underline{C}_{4}$ & $\underline{C}_{5}$ & $\underline{C}_{6}$ & $\underline{C}_{7}$ & $\underline{C}_{8}$ \\
\hline 0.00 & 0.44 & 0.87 & 1.31 & 1.74 & 2.18 & 2.62 & 3.05 & 3.49 \\
\hline 0.05 & 0.38 & 0.76 & 1.15 & 1.54 & 1.93 & 2.32 & 2.70 & \\
\hline 0.10 & 0.33 & 0.69 & 1.05 & 1.41 & 1.77 & 2.12 & & \\
\hline 0.15 & 0.28 & 0.64 & 0.97 & 1.30 & 1.60 & & & \\
\hline \multicolumn{9}{|c|}{$\pi(N)=4 \times I\{N \leq 4\}$} \\
\hline & \multicolumn{8}{|c|}{ Entry Thresholds } \\
\hline$\sigma$ & $\bar{C}_{1}$ & $\bar{C}_{2}$ & $\bar{C}_{3}$ & $\bar{C}_{4}$ & $\bar{C}_{5}$ & $\bar{C}_{6}$ & $\bar{C}_{7}$ & $\bar{C}_{8}$ \\
\hline 0.00 & 0.50 & 1.00 & 1.50 & 1.99 & & & & \\
\hline 0.05 & 0.48 & 0.98 & 1.52 & 1.91 & & & & \\
\hline 0.10 & 0.46 & 1.00 & 1.57 & 1.78 & & & & \\
\hline 0.15 & 0.46 & 1.04 & 1.56 & 1.77 & & & & \\
\hline
\end{tabular}

Exit Thresholds

\begin{tabular}{ccccccccc}
$\sigma$ & $\underline{C}_{1}$ & $\underline{C}_{2}$ & $\underline{C}_{3}$ & $\underline{C}_{4}$ & $\underline{C}_{5}$ & $\underline{C}_{6}$ & $\underline{C}_{7}$ & $\underline{C}_{8}$ \\
\hline 0.00 & 0.44 & 0.87 & 1.31 & 1.74 & & & & \\
0.05 & 0.38 & 0.76 & 1.16 & 1.51 & & & & \\
0.10 & 0.33 & 0.69 & 1.05 & 1.31 & & & & \\
0.15 & 0.28 & 0.64 & 0.96 & 1.19 & & & &
\end{tabular}

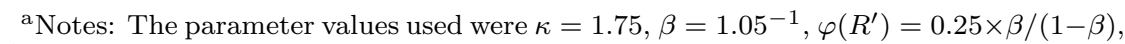
$\hat{C}=e^{-1.5}, \check{C}=e^{1.5}$, and $Q(\cdot \mid C)$ a mixture over reflected random walks in the logarithm of $C$ with uniformly distributed innovations and approximate innovation variance $\sigma^{2}$. An empty cell indicates that the ergodic distribution of $N_{t}$ puts zero probability on the given value of $N$. Please see the text for further details. 
Table 2: Static Probit Analysis of Market Structure ${ }^{\mathrm{a}}$

\begin{tabular}{|c|c|c|c|c|c|c|c|c|}
\hline & \multicolumn{8}{|c|}{ Implied Static Entry Thresholds } \\
\hline$\sigma$ & $C_{1}^{\star}$ & $C_{2}^{\star}$ & $C_{3}^{\star}$ & $C_{4}^{\star}$ & $C_{5}^{\star}$ & $C_{6}^{\star}$ & $C_{7}^{\star}$ & $C_{8}^{\star}$ \\
\hline 0.00 & 0.44 & 0.93 & 1.37 & 1.86 & 2.37 & 2.95 & 3.41 & 3.90 \\
\hline 0.05 & 0.43 & 0.87 & 1.32 & 1.78 & 2.24 & 2.70 & 3.13 & \\
\hline 0.10 & 0.39 & 0.83 & 1.29 & 1.75 & 2.20 & 2.62 & & \\
\hline \multirow[t]{2}{*}{0.15} & 0.34 & 0.81 & 1.26 & 1.70 & 2.05 & & & \\
\hline & \multicolumn{8}{|c|}{ Implied $\pi(N) / \pi(1)$ for $N=$} \\
\hline$\sigma$ & 1 & 2 & 3 & 4 & 5 & 6 & 7 & 8 \\
\hline 0.00 & 1.00 & 0.95 & 0.97 & 0.95 & 0.93 & 0.90 & 0.91 & 0.91 \\
\hline 0.05 & 1.00 & 0.98 & 0.97 & 0.96 & 0.95 & 0.95 & 0.95 & \\
\hline 0.10 & 1.00 & 0.94 & 0.92 & 0.90 & 0.89 & 0.90 & & \\
\hline 0.15 & 1.00 & 0.85 & 0.82 & 0.81 & 0.83 & & & \\
\hline
\end{tabular}

a Notes: The table's top panel reports population values of Probit-based entry thresholds from the static model of Bresnahan and Reiss calculated using the ergodic distribution of the dynamic model specification of Section 4.2 and Table 1 . The bottom panel reports the implied values of $\pi(N)$ normalized by $\pi(1)$. An empty cell indicates that the ergodic distribution of $N_{t}$ puts zero probability on the given value of $N$. Please see the text for further details.

Given observations of $C$ and $N$ from a cross section of markets, ordered Probit estimation immediately yields estimates of $C_{1}^{\star}, \ldots, C_{N}^{\star}$; and $\varsigma$. With these, Bresnahan and Reiss estimate how the producer surplus per consumer falls with additional competitors. Specifically, the definition of $C_{N}^{\star}$ implies that $\pi(N) / \pi(1)=$ $C_{1}^{\star} \times N / C_{N}^{\star}$. If the level of demand required to support $N$ firms equals $N$ times the level required for a monopolist, then the producer surplus per consumer does not fall with additional entry. On the other hand, if demand must exceed $N \times C_{1}^{\star}$ to induce $N$ firms to enter, then the surplus per consumer must decline with $N$. In this way, Bresnahan and Reiss infer the toughness of competition from the relationship between market size and the number of competitors.

For any given joint distribution of $C$ and $N$, we can define the population counterparts to the estimated thresholds by minimizing the population analogue of the ordered Probit's log-likelihood function,

$$
\mathcal{L}(\cdot) \equiv \mathbb{E}\left[\sum_{n=0}^{\check{N}} I\{N=n\} \ln \left(\Phi\left(\frac{\ln \left(C / C_{n}^{\star}\right)}{\varsigma}\right)-\Phi\left(\frac{\ln \left(C / C_{n+1}^{\star}\right)}{\varsigma}\right)\right)\right] .
$$

Because the ordered Probit likelihood function is always concave, even if it does not represent the true data generating process, this function is minimized at a unique set of static entry thresholds $C_{1}^{\star}, \ldots, C_{N}^{\star}$. Calculating these static entry thresholds using the joint distribution of $C_{t}$ and $N_{t}$ from the fully dynamic model's ergodic distribution, mimicking Bresnahan and Reiss by using these to "estimate" $\pi(N) / \pi(1)$, and comparing these "estimates" to the true values of $\pi(N) / \pi(1)$ from the dynamic model indicates whether static estimates of the toughness of competition suffer from substantial bias.

The top panel of Table 2 reports population values of the Probit-based static entry thresholds $C_{1}^{\star}, \ldots, C_{\tilde{N}}^{\star}$ calculated using the ergodic distribution of the dynamic model specification examined in Section 4.2. Its bottom panel gives the implied "estimates" of $\pi(N) / \pi(1)$. For all three of values of $\sigma$ used, the static entry thresholds almost exactly equal the average of the dynamic model's corresponding entry and exit thresholds. That is, the static analysis "splits the difference" between them.

Recall that the true values of $\pi(N) / \pi(1)$ all equal one. That is, an additional competitor steals business from incumbents but does not lower the producer surplus earned per consumer. For the case with $\sigma=$ 0.05 , the implied values deviate little from the truth. However, raising $\sigma$ further substantially lowers these "estimates". When $\sigma=0.15$, the implied value of $\pi(2) / \pi(1)$ equals 0.85 . Further increases in $N$ change this little. 
Apparently, the static Probit analysis can find evidence that $\pi(N)$ falls with data generated from a dynamic model in which $\pi(N)$ is constant. To gain some insight into how sunk costs and uncertainty affect the static analysis of the toughness of competition, recall that the static Probit's thresholds are roughly the average of the dynamic entry and exit thresholds. Hence, without uncertainty both the static and the dynamic thresholds are evenly spaced if $\pi(N)$ is constant. For example, from the static analysis we may find that it takes 1000 consumers for one firm and 2000 consumers for two firms to be active in the market. With uncertainty, however, option value considerations will make firms of all ranks more reluctant to exit. Because this effect is not offset by a change in the entry thresholds (see the previous section and Table 1), the static thresholds will decrease as well. We may now "observe" that it takes only 500 consumers for one firm and 1500 consumers for two firms to be active. The fact that the number of consumers needs to triple to entice a second firm to join the first suggests that the producer surplus per consumer served falls substantially when a second firm enters. However, this inference arises from uncertainty; the actual producer surplus per consumer served is constant. ${ }^{16}$

In our analysis, the delay in exit arising from option-value considerations imparts a substantial downward bias to each estimated threshold. This bias is large in the specification under consideration. Determining its importance for empirical work must proceed on a case-by-case basis, but we expect option-value considerations to pervade oligopolists' exit decisions. A comparison of the results of Bresnahan and Reiss (1991b) with the "estimates" in Table 2 supports this view. Their abstract reports:

Our empirical results suggest that competitive conduct changes quickly as the number of incumbents increases. In markets with five or fewer incumbents, almost all variation in competitive conduct occurs with the entry of the second or third firm.

This is exactly the pattern displayed in Table 2 .

\section{A Proofs of Results in Section 2}

Proof of Proposition 1. The proof proceeds by first constructing a candidate equilibrium strategy and then verifying that it is a LIFO strategy that forms an equilibrium and satisfies the proposition's requirements.

Preliminaries Define $\check{N}$ as in Section 4.1. Because $\check{C}$ is finite, $\pi(N)$ is weakly decreasing in $N$, and $\kappa>0$; $\check{N}<\infty$. This is an upper bound on the number of firms that would ever produce in a LIFO equilibrium.

Next, consider the exit decision problem of a firm with (prospective) rank $R^{\prime} \leq \check{N}$ that expects the number of firms to evolve according to the deterministic transition rule $N_{R^{\prime}}^{\prime}: \mathbb{Z}_{+} \times[\overline{\hat{C}}, \check{C}] \rightarrow\left\{R^{\prime}, R^{\prime}+1, \ldots, \check{N}\right\}$. Here, $N_{R^{\prime}}^{\prime}(X, C)$ is the number of firms that the firm with rank $R^{\prime}$ expects to be active next period given a decision to continue, that $X$ younger firms are active this period, and that the number of consumers equals $C$. The number of active firms next period is defined for the event that $R^{\prime}+X>\check{N}$, but it never exceeds $\check{N}$. Define $\mathcal{W}$ to be the space of all functions

$$
w:\{0, \ldots, \check{N}-1\} \times[\hat{C}, \check{C}] \rightarrow\left[0, \frac{\beta \pi(1) \check{C}}{1-\beta}\right]
$$

and define the Bellman operator $T_{R^{\prime}}: \mathcal{W} \rightarrow \mathcal{W}$ with

$$
T_{R^{\prime}}(w)(X, C)=\max _{a \in[0,1]} a \beta \mathbb{E}\left[\frac{\pi\left(N_{R^{\prime}}^{\prime}(X, C)\right) C^{\prime}}{N_{R^{\prime}}^{\prime}(X, C)}-\kappa+w\left(N_{R^{\prime}}^{\prime}(X, C)-R^{\prime}, C^{\prime}\right)\right] .
$$

Note that $T_{R^{\prime}}$ depends on the specification for $N_{R^{\prime}}^{\prime}$. It satisfies Blackwell's sufficient conditions for a contraction mapping, and $\mathcal{W}$ is a complete metric space. Hence, $T_{R^{\prime}}$ has a unique fixed point, the value function $w_{R^{\prime}}$.

\footnotetext{
${ }^{16}$ Another logical possibility is that the fall in the implied static thresholds reflects mean reversion: Because $C$ cannot fall below $\hat{C}$, a potential entrant does not expect extremely low values of $C$ to persist. We examined whether this contributes to our results by changing $\hat{C}$ and $\breve{C}$ from $e^{-1.5}$ and $e^{1.5}$ to $e^{-2}$ and $e^{2}$. The results differ only minimally from those in Table 2 .
} 
Construction of a candidate equilibrium Begin with the decision problem for a firm with rank $\check{N}$ and the transition rule $N_{\tilde{N}}^{\prime}(X, C)=\check{N}$ for all $(X, C)$. This transition rule reflects the firm's expectations that it produces no longer than any earlier entrant, any younger active firms will exit, and no firms will enter. The fixed point $w_{\tilde{N}}$ of $T_{\check{N}}$ can be uniquely extended to a value function on the entire state space $\mathbb{Z}_{+} \times[\hat{C}, \check{C}]$ by assigning $w_{\check{N}}(X, C)=w_{\check{N}}(0, C)$ for all $(X, C)$. Denote $\left\{C \mid w_{\check{N}}(0, C)>0\right\}$ with $\mathcal{S}_{\check{N}}$ and $\left\{C \mid w_{\check{N}}(0, C)>\varphi(\check{N})\right\}$ with $\mathcal{E}_{\check{N}}$. Note that $\mathcal{S}_{\check{N}} \supseteq \mathcal{E}_{\check{N}}$. Under the maintained hypotheses of this maximization problem, this firm chooses to remain active if and only if $C \in \mathcal{S}_{\check{N}}$ and it chooses to enter the industry if and only if $C \in \mathcal{E}_{\check{N}^{\prime}}{ }^{17}$

Next, iterate the following argument for $R^{\prime}=\check{N}-1, \ldots, 1$.

(i). Suppose that we have in hand value functions $w_{R^{\prime}+1}, \ldots, w_{\check{N}}$; entry sets $\mathcal{E}_{R^{\prime}+1}, \ldots, \mathcal{E}_{\check{N}}$; and survival sets $\mathcal{S}_{R^{\prime}+1}, \ldots, \mathcal{S}_{\tilde{N}} ;$ and that

$$
\begin{aligned}
& -\mathcal{E}_{R^{\prime}+1} \supseteq \cdots \supseteq \mathcal{E}_{\check{N}}, \\
& -\mathcal{S}_{R^{\prime}+1} \supseteq \cdots \supseteq \mathcal{S}_{\check{N}}, \\
& -\mathcal{S}_{\tilde{R}} \supseteq \mathcal{E}_{\tilde{R}} \text { for all } \tilde{R} \geq R^{\prime}+1, \text { and } \\
& -w_{\tilde{R}}(X, C)=w_{\tilde{R}}(\check{N}-\tilde{R}, C) \text { if } X>\check{N}-\tilde{R} \text { for all } \tilde{R} \geq R^{\prime}+1 .
\end{aligned}
$$

(ii). Consider the decision problem for a firm with rank $R^{\prime}$ and transition rule

$$
N_{R^{\prime}}^{\prime}(X, C)=R^{\prime}+\sum_{i=1}^{\infty}\left[I\left\{i \leq X, C \in \mathcal{S}_{R^{\prime}+i}\right\}+I\left\{i>X, C \in \mathcal{E}_{R^{\prime}+i}\right\}\right]
$$

where $\mathcal{E}_{\tilde{R}}=\mathcal{S}_{\tilde{R}}=\emptyset$ for $\tilde{R}>\check{N}$. This transition rule reflects the firm's expectations that it produces no longer than any earlier entrant and that $\mathcal{E}_{R^{\prime}+i}$ and $\mathcal{S}_{R^{\prime}+i}, i \in \mathbb{N}$, govern younger firms' entry and survival. The specification for $N_{R^{\prime}}^{\prime}$ implies that $N_{R^{\prime}}^{\prime}(X, C)=N_{R^{\prime}}^{\prime}\left(\check{N}-R^{\prime}, C\right)$ if $X>\check{N}-R^{\prime}$. Therefore, we can uniquely extend the fixed point $w_{R^{\prime}}$ of $T_{R^{\prime}}$ to a value function on the entire state space $\mathbb{Z}_{+} \times[\hat{C}, \check{C}]$ by assigning $w_{R^{\prime}}(X, C)=w_{R^{\prime}}\left(\check{N}-R^{\prime}, C\right)$ for all $X>\check{N}-R^{\prime}$.

(iii). Denote the complete subspace of functions $w$ such that

$$
\begin{aligned}
& -w(X+1, C) \geq w_{R^{\prime}+1}(X, C), X=0, \ldots, \check{N}-2, \text { and } \\
& -w(X, C) \text { is weakly decreasing in } X, \text { for all } C ;
\end{aligned}
$$

with $\mathcal{W}_{R^{\prime}} \subseteq \mathcal{W}$. We wish to show that $T_{R^{\prime}}$ maps $\mathcal{W}_{R^{\prime}}$ into itself. Because $\mathcal{S}_{\tilde{R}} \supseteq \mathcal{E}_{\tilde{R}}$ for all $\tilde{R} \geq R^{\prime}+1$ by supposition, Equation (3) implies that $N_{R^{\prime}+1}^{\prime}(X, C) \geq N_{R^{\prime}}^{\prime}(X+1, C)$, so

$$
\frac{\pi\left(N_{R^{\prime}}^{\prime}(X+1, C)\right) C^{\prime}}{N_{R^{\prime}}^{\prime}(X+1, C)} \geq \frac{\pi\left(N_{R^{\prime}+1}^{\prime}(X, C)\right) C^{\prime}}{N_{R^{\prime}+1}^{\prime}(X, C)}
$$

and for $w \in \mathcal{W}_{R^{\prime}}$

$$
\begin{aligned}
w\left(N_{R^{\prime}}^{\prime}(X+1, C)-R^{\prime}, C^{\prime}\right) & \geq w\left(N_{R^{\prime}+1}^{\prime}(X, C)-\left(R^{\prime}+1\right)+1, C^{\prime}\right) \\
& \geq w_{R^{\prime}+1}\left(N_{R^{\prime}+1}^{\prime}(X, C)-\left(R^{\prime}+1\right), C^{\prime}\right) .
\end{aligned}
$$

(iv). For $w \in \mathcal{W}_{R^{\prime}}$, applying these inequalities to the Bellman operator bounds $T_{R^{\prime}}(w)(X+1, C)$ from below by $w_{R^{\prime}+1}(X, C)$ because

\footnotetext{
${ }^{17}$ This specification of $\mathcal{S}_{\check{N}}$ and $\mathcal{E}_{\check{N}}$ ensures that the firm defaults to inactivity in the case of indifference.
} 


$$
\begin{aligned}
& \max _{a \in[0,1]} a \beta \mathbb{E}\left[\frac{\pi\left(N_{R^{\prime}}^{\prime}(X+1, C)\right) C^{\prime}}{N_{R^{\prime}}^{\prime}(X+1, C)}-\kappa+w\left(N_{R^{\prime}}^{\prime}(X+1, C)-R^{\prime}, C^{\prime}\right)\right] \\
& \geq \max _{a \in[0,1]} a \beta \mathbb{E}\left[\frac{\pi\left(N_{R^{\prime}+1}^{\prime}(X, C)\right) C^{\prime}}{N_{R^{\prime}+1}^{\prime}(X, C)}-\kappa+w_{R^{\prime}+1}\left(N_{R^{\prime}+1}^{\prime}(X, C)-\left(R^{\prime}+1\right), C^{\prime}\right)\right] \\
& =w_{R^{\prime}+1}(X, C) .
\end{aligned}
$$

The transition rule $N_{R^{\prime}}^{\prime}(X, C)$ weakly increases in $X$, so that $T_{R^{\prime}}(w)(X, C)$ weakly decreases in $X$ if $w \in \mathcal{W}_{R^{\prime}}$. Therefore, $w \in \mathcal{W}_{R^{\prime}} \Longrightarrow T_{R^{\prime}}(w) \in \mathcal{W}_{R^{\prime}}$. Since $T_{R^{\prime}}$ is a contraction mapping and $\mathcal{W}_{R^{\prime}}$ is complete, $T_{R^{\prime}}$ has a unique fixed point $w_{R^{\prime}} \in \mathcal{W}_{R^{\prime}}$. These properties extend to the entire state space $\mathbb{Z}_{+} \times[\hat{C}, \check{C}]$; because, for $X \geq \check{N}-R^{\prime}$,

$$
\begin{aligned}
& -w_{R^{\prime}}(X+1, C)=w_{R^{\prime}}\left(\check{N}-R^{\prime}, C\right) \geq w_{R^{\prime}+1}\left(\check{N}-R^{\prime}-1, C\right)=w_{R^{\prime}+1}(X, C) \text { and } \\
& -w_{R^{\prime}}(X, C)=w_{R^{\prime}}\left(\check{N}-R^{\prime}, C\right) .
\end{aligned}
$$

(v). Finish this step of the recursion by noting that $w_{R^{\prime}}(0, C) \geq w_{R^{\prime}}(1, C) \geq w_{R^{\prime}+1}(0, C)$ for all $C$, so $\mathcal{E}_{R^{\prime}} \equiv\left\{C \mid w_{R^{\prime}}(0, C)>\varphi\left(R^{\prime}\right)\right\} \supseteq \mathcal{E}_{R^{\prime}+1}, \mathcal{S}_{R^{\prime}} \equiv\left\{C \mid w_{R^{\prime}}(0, C)>0\right\} \supseteq \mathcal{S}_{R^{\prime}+1}$, and $\mathcal{S}_{R^{\prime}} \supseteq \mathcal{E}_{R^{\prime}}$.

With the recursion complete, specify the candidate equilibrium strategy as

$$
\begin{aligned}
A_{S}\left(X, C, R^{\prime}\right) & = \begin{cases}1 & \text { if } C \in \mathcal{S}_{R^{\prime}} \text { and } \\
0 & \text { otherwise }\end{cases} \\
A_{E}\left(C, R^{\prime}\right) & = \begin{cases}1 & \text { if } C \in \mathcal{E}_{R^{\prime}} \text { and } \\
0 & \text { otherwise }\end{cases}
\end{aligned}
$$

Verification Since $A_{S}\left(X, C, R^{\prime}\right)$ is constant in $X$, the candidate is a LIFO strategy. The candidate is pure, and it weakly decreases in $R^{\prime}$ since $\mathcal{S}_{R^{\prime}} \supseteq \mathcal{S}_{R^{\prime}+1}$ and $\mathcal{E}_{R^{\prime}} \supseteq \mathcal{E}_{R^{\prime}+1}$. Finally, $A_{S}\left(X, C, R^{\prime}\right) \geq A_{E}\left(C, R^{\prime}\right)$, because $\mathcal{S}_{R^{\prime}} \supseteq \mathcal{E}_{R^{\prime}}$. Hence, the candidate equilibrium strategy satisfies the proposition's stated requirements.

It forms a symmetric Markov-perfect equilibrium if no firm can gain by a one-shot deviation from it (e.g. Fudenberg and Tirole, 1991, Theorem 4.2). The value to an active firm if all firms follow the strategy is $v\left(X, C, R^{\prime}\right)=w_{R^{\prime}}(X, C)$. By construction, the strategy dictates an optimal action for entrants and for active firms contemplating the period's final survival decision (i.e. $X=0$ ). To show that the strategy also dictates an optimal action for an active firm facing $X \geq 1$ active firms with higher names, note that survival is optimal if $C \in \mathcal{S}_{\mathcal{R}^{\prime}}$ because either $C \notin \mathcal{S}_{R^{\prime}+1}$, so that $w_{R^{\prime}}(X, C)=w_{R^{\prime}}(0, C)>0$, or $C \in \mathcal{S}_{R^{\prime}+1}$, so that $w_{R^{\prime}}(X, C) \geq w_{R^{\prime}+1}(X-1, C)>0$. If instead $C \notin \mathcal{S}_{R^{\prime}}$, then exit is optimal because $w_{R^{\prime}}(X, C) \leq w_{R^{\prime}}(0, C) \leq$ 0 . Therefore, no firm can profit from a one-shot deviation and the strategy forms an equilibrium.

Proof of Proposition 2. The LIFO strategy constructed in the proof of Proposition 1 defaults to inactivity. Thus, a symmetric Markov-perfect equilibrium in a LIFO strategy that defaults to inactivity exists.

Uniqueness can be proven recursively, following the recursive construction of a candidate equilibrium strategy in the proof of Proposition 1.

(i). In any equilibrium in a LIFO strategy that defaults to inactivity, the expected discounted profits $v\left(X, C, R^{\prime}\right)$ equal 0 and the entry and survival sets equal $\mathcal{E}_{R^{\prime}}=\mathcal{S}_{R^{\prime}}=\emptyset$ in all states $\left(X, C, R^{\prime}\right)$ such that $R^{\prime}>\check{N}$.

(ii). Consequently, in any such equilibrium, $N_{\tilde{N}}^{\prime}(X, C)$ gives the number of firms in the next period in all states $(X, C, \Sigma)$. Therefore, the expected discounted profits $v(X, C, \check{N})$ equal the unique solution $w_{\check{N}}(X, C)$ to Proposition 1's decision problem of a firm with rank $\check{N}$ in all states $(X, C, \check{N})$. The equilibrium entry set equals this decision problem's entry set $\mathcal{E}_{\check{N}}$, and the equilibrium survival set equals its survival set $\mathcal{S}_{\check{N}}$. 
(iii). Next, iterate the following argument for $R^{\prime}=\check{N}-1, \ldots, 1$. Suppose that, in any equilibrium in a LIFO strategy that defaults to inactivity, the expected continuation value $v(X, C, \tilde{R})$ equals $w_{\tilde{R}}(X, C)$, the entry set equals $\mathcal{E}_{\tilde{R}}$, and the survival set equals $\mathcal{S}_{\tilde{R}}$ in all states $(X, C, \tilde{R})$ such that $\tilde{R}>R^{\prime}$. Then, $N_{R^{\prime}}^{\prime}(X, C)$ defined by equation (3) gives the number of firms in the next period in state $\left(X, C, R^{\prime}\right)$. Hence, in all such equilibria, the expected continuation value $v\left(X, C, R^{\prime}\right)$ equals the solution $w_{R^{\prime}}(X, C)$ to Proposition 1's decision problem of a firm with rank $R^{\prime}$ in all states $\left(X, C, R^{\prime}\right)$. The equilibrium entry set equals this decision problem's entry set $\mathcal{E}_{R^{\prime}}$, and the equilibrium survival set equals its survival set $\mathcal{S}_{R^{\prime}}$.

(iv). Recall from Proposition 1's proof that the equilibrium survival rule $A_{S}\left(X, C, R^{\prime}\right)$ is constant in $X$ and weakly decreases with $R^{\prime}$; and the equilibrium entry rule $A_{E}\left(C, R^{\prime}\right)$ weakly decreases with $R^{\prime}$.

\section{B Proofs of Results in Section 3}

Proof of Proposition 3. The strategy of Proposition 2's equilibrium can be written as $A_{S}\left(N-R, C, R^{\prime}\right)=$ $I\left\{C \in \mathcal{S}_{R^{\prime}}\right\}$ and $A_{E}\left(C, R^{\prime}\right)=I\left\{C \in \mathcal{E}_{R^{\prime}}\right\}$, where the sets $\mathcal{S}_{R^{\prime}}$ and $\mathcal{E}_{R^{\prime}}$ are those defined in the proof of Proposition 1. From that proof, we know that $\mathcal{E}_{1} \supseteq \cdots \supseteq \mathcal{E}_{\check{N}}, \mathcal{S}_{1} \supseteq \cdots \supseteq \mathcal{S}_{\check{N}}$, and $\mathcal{S}_{R^{\prime}} \supseteq \mathcal{E}_{R^{\prime}}$ for $R^{\prime}=1, \cdots, \check{N}$.

Threshold rules imply monotonicity Suppose that firms of all ranks follow threshold strategies, and denote the entry and exit thresholds with $\bar{C}_{1}, \bar{C}_{2}, \ldots, \bar{C}_{\check{N}}$ and $\underline{C}_{1}, \underline{C}_{2}, \ldots, \underline{C}_{\check{N}}$. From the above properties of the equilibrium strategy, we know that $\bar{C}_{1} \leq \cdots \leq \bar{C}_{\check{N}}, \underline{C}_{1} \leq \cdots \leq \underline{C}_{\check{N}}$, and $\underline{C}_{R^{\prime}} \leq \bar{C}_{R^{\prime}}$ for $R^{\prime}=1, \cdots, \check{N}$. This allows us to follow Equation (3) and write the transition rule for $N_{t}$ as

$$
N_{t+1}=\sum_{j=1}^{\infty}\left[I\left\{j \leq N_{t}, C>\underline{C}_{j}\right\}+I\left\{j>N_{t}, C>\bar{C}_{j}\right\}\right],
$$

In this, we set $\bar{C}_{i}=\check{C}$ for $i>\check{N}$. This is obviously increasing in $C_{t}$, so the proposition is true for the case with $i=1$. To show that this is true for higher $i$, proceed inductively. Suppose that $C_{t}$ weakly increases $N_{t+i-1}$. Inspection of (4) shows that increasing $N_{t+i-1}$ weakly increases $N_{t+i}$, so increasing $C_{t}$ weakly increases $N_{t+i}$.

Monotonicity implies threshold rules Suppose that all firms' entry and exit rules are such that increasing $C_{t}$ weakly increases $N_{t+i}$ for positive $i$ and any possible initial condition $\left(N_{t}, C_{t}\right)$ and sequence of subsequent demand realizations $C_{t+1}, \ldots, C_{t+i-1}$. To show that this implies that all firms' entry and exit decisions follow threshold policies, suppose to the contrary that the survival decision of a firm with some rank $R^{\prime}$ does not. That is, there exist two values of $C_{t}, C_{L}<C_{H}$ such that $C_{L} \in \mathcal{S}_{R^{\prime}}$ and $C_{H} \notin \mathcal{S}_{R^{\prime}}$. Suppose that $N_{t}=R^{\prime}$ and increase $C_{t}$ from $C_{L}$ to $C_{H}$. Then, this change decreases $N_{t+1}$ by at least one firm (the decrease is two or more if $C_{L} \in \mathcal{E}_{R^{\prime}+1}$ or $C_{H} \notin \mathcal{S}_{R^{\prime}-1}$ ). This contradicts the original supposition, so all firms' survival decisions must follow threshold rules.

Next, suppose that the entry of a firm with potential rank $R^{\prime}$ does not follow a threshold rule. Again there must exist $C_{L}<C_{H}$ such that $C_{L} \in \mathcal{E}_{R^{\prime}}$ and $C_{H} \notin \mathcal{E}_{R^{\prime}}$. Suppose that $N_{t}=R^{\prime}-1$. Because $\mathcal{S}_{R^{\prime}-1} \supseteq \mathcal{S}_{R^{\prime}} \supseteq \mathcal{E}_{R^{\prime}}$, all $R^{\prime}-1$ incumbent firms continue if $C_{t}=C_{L}$. Thus, increasing $C_{t}$ from $C_{L}$ to $C_{H}$ reduces $N_{t+1}$ by at least one firm (the decrease is two or more if $C_{L} \in \mathcal{E}_{R^{\prime}+1}$ or $C_{H} \notin \mathcal{S}_{R^{\prime}-1}$ ), which again contradicts the original supposition. Hence threshold policies govern the survival and entry decisions of firms of all ranks.

The proof of Proposition 4 relies on Proposition 5, so we first present a proof of the latter.

Proof of Proposition 5. The proof has two parts. 


\section{The proposition's mixture representation implies Assumptions 1-3}

(i). Assumption 1 is satisfied, because $\mu_{k}^{K}(C)$ is nondecreasing with $C$ for all $k, K ; \mathbb{E}\left[C^{\prime} \mid C\right]$ equals $\mu(C) \equiv$ $\lim _{K \rightarrow \infty} \sum_{k=1}^{K} p_{k}^{K} \mu_{k}^{K}(C)$ by the bounded convergence theorem (Billingsley, 1995, Theorem 16.5); and the set of nondecreasing functions is closed.

(ii). Define $\widetilde{Q}_{k}^{K}(u \mid C) \equiv Q_{k}^{K}\left(u+\mu_{k}^{K}(C) \mid C\right)$ and denote the distribution of $U^{\prime}$ given $C$ with $\widetilde{Q}(u \mid C) \equiv Q(u+$ $\mu(C) \mid C)$. The functions $\widetilde{Q}_{k}^{K}(\cdot \mid C) ; k=1, \ldots, K ; K \in \mathbb{N}$; do not depend on $C$; and the presumption that $Q(\cdot \mid C)$ has the proposition's mixture representation requires that $\widetilde{Q}(\cdot \mid C)=\lim _{K \rightarrow \infty} \sum_{k=1}^{K} p_{k}^{K} \widetilde{Q}_{k}^{K}(\cdot \mid C)$. Therefore, Assumption 2 is satisfied.

(iii). Define $\widehat{Q}_{k}^{K}(u \mid C) \equiv Q_{k}^{K}(u+\mu(C) \mid C)$. From the proposition's final condition,

$$
\mu_{k}^{K}(C)-\sigma_{k}^{K} / 2-\mu(C) \leq C-\mu(C) .
$$

Consequently, $\widehat{Q}_{k}^{K}(\cdot \mid C)$ is a uniform distribution with support

$$
\left[\mu_{k}^{K}(C)-\sigma_{k}^{K} / 2-\mu(C), \mu_{k}^{K}(C)+\sigma_{k}^{K} / 2-\mu(C)\right]
$$

that includes or lies below $C-\mu(C)$. Now, the presumption that $Q(\cdot \mid C)$ has the proposition's mixture representation requires that

$$
\widetilde{Q}(\cdot \mid C)=\lim _{K \rightarrow \infty} \sum_{k=1}^{K} p_{k}^{K} \widehat{Q}_{k}^{K}(u \mid C) .
$$

By a result of A.I. Khintchine (Feller, 1971, p. 158), this implies that $\widetilde{Q}(\cdot \mid C)$ is concave on $[C-\mu(C), \infty)$. We have already shown that Assumption 2 holds good, so that $\widetilde{Q}(\cdot \mid C)$ equals $\widetilde{Q}(\cdot)$, and is concave above $C-\mu(C)$ for all C. Because $\mu(C)=\mathbb{E}\left[C^{\prime} \mid C\right]$, this implies Assumption 3 .

Assumptions 1-3 imply the proposition's mixture representation The construction proceeds in two steps:

(i). We write the distribution $\widetilde{Q}$ corresponding to $Q(\cdot \mid C)$ as a mixture of uniform distributions that are independent of $C$, distinguishing between its unrestricted part on $(-\infty, \underline{U})$ and its concave part on $[\underline{U}, \infty)$.

(ii). Subsequently, we write $Q(\cdot \mid C)$ as this same mixture, with $\mathbb{E}\left[C^{\prime} \mid C\right]$ added to the mean of each uniform distribution, and verify that the construction satisfies the requirements of the proposition.

Let $G_{\mu, \sigma}$ denote a uniform cumulative distribution function on $[\mu-\sigma / 2, \mu+\sigma / 2]$. In particular, $G_{\mu, 0}$ denotes a distribution that is degenerate at $\mu$.

(i). Consider the construction of $\widetilde{Q}$ as a mixture of uniform distributions.

(a) First, consider the restriction of $\widetilde{Q}$ to $(-\infty, \underline{U})$. Fix an arbitrary $\delta>0$. By the Jordan decomposition theorem (Feller, 1971, p. 138), $\widetilde{Q}$ can be written as a mixture of a discrete and a continuous distribution function. Both the discrete and the continuous part are nondecreasing and bounded on $(-\infty, \underline{U})$. This ensures that there exist

- a finite (and possibly defective) mixture of degenerate distributions with supports in $(-\infty, \underline{U})$ that is within a $\delta / 2$ uniform distance from the discrete part; and

- a piecewise-linear (and possibly defective) cumulative distribution function with support on a closed subset of $(-\infty, \underline{U})$ that uniformly approximates the continuous part up to a $\delta / 2$ distance. 
Because the latter can be written as a finite mixture of uniform distributions with supports in $(-\infty, \underline{U})$, it follows from the triangle inequality that $\widetilde{Q}$ on $(-\infty, \underline{U})$ can be approximated up to a $\delta$ uniform distance by a finite mixture of, possibly degenerate, uniform distributions $G_{\mu, \sigma}$. Because $\delta>0$ was arbitrary, this implies that there exist $p_{k}^{K} \geq 0, \sigma_{k}^{K} \geq 0$, and $\nu_{k}^{K}<\underline{U}-\sigma_{k}^{K} / 2$; $k=1, \ldots, K / 2$; such that $\sum_{k=1}^{K / 2} p_{k}^{K}=\widetilde{Q}\left(\underline{U}^{-}\right) \equiv \lim _{u \uparrow \underline{U}} \widetilde{Q}(u)$, and

$$
\lim _{K \rightarrow \infty} \sup _{u<\underline{U}}\left|\widetilde{Q}(u)-\sum_{k=1}^{K / 2} p_{k}^{K} G_{\nu_{k}^{K}, \sigma_{k}^{K}}(u)\right|=0,
$$

for even $K \in \mathbb{N}$.

(b) Because $\widetilde{Q}$ is concave on $[\underline{U}, \infty)$ (Assumption 3), by a result of A.I. Khintchine (Feller, 1971, p. 158), there exist $p_{k}^{K} \geq 0, \sigma_{k}^{K} \geq 0$, and $\nu_{k}^{K} \equiv \underline{U}+\sigma_{k}^{K} / 2 ; k=K / 2+1, \ldots, K$; such that $\sum_{k=K / 2+1}^{K} p_{k}^{K}=1-\widetilde{Q}\left(\underline{U}^{-}\right)$, and

$$
\lim _{K \rightarrow \infty} \sup _{u \geq \underline{U}}\left|\widetilde{Q}(u)-\widetilde{Q}\left(\underline{U}^{-}\right)-\sum_{k=K / 2+1}^{K} p_{k}^{K} G_{\nu_{k}^{K}, \sigma_{k}^{K}}(u)\right|=0,
$$

for even $K \in \mathbb{N}$.

(ii). From (5), (6), and the triangle inequality, it follows that

$$
\lim _{K \rightarrow \infty} \sup _{u}\left|\widetilde{Q}(u)-\sum_{k=1}^{K} p_{k}^{K} G_{\nu_{k}^{K}, \sigma_{k}^{K}}(u)\right|=0
$$

for even $K \in \mathbb{N}$. Define $\mu_{k}^{K}(C) \equiv \nu_{k}^{K}+\mathbb{E}\left[C^{\prime} \mid C\right]$ and $Q_{k}^{K}(\cdot \mid C) \equiv G_{\mu_{k}^{K}(C), \sigma_{k}^{K}}(\cdot)$. From (7), we have

$$
\lim _{K \rightarrow \infty} \sup _{c, C}\left|Q(c \mid C)-\sum_{k=1}^{K} p_{k}^{K} Q_{k}^{K}(c \mid C)\right|=0,
$$

for even $K \in \mathbb{N}$. Moreover, each $\mu_{k}^{K}(C)$ is weakly increasing with $C$ and $Q_{k}^{K}(\cdot \mid C)$ is a uniform distribution with the lower end of its support equal to

$$
\nu_{k}^{K}+\mathbb{E}\left[C^{\prime} \mid C\right]-\sigma_{k}^{K} / 2<\underline{U}+\mathbb{E}\left[C^{\prime} \mid C\right]-\sigma_{k}^{K} / 2 \leq C-\sigma_{k}^{K} / 2 \leq C
$$

if $k \leq K / 2$, and equal to $\underline{U}+\mathbb{E}\left[C^{\prime} \mid C\right] \leq C$ if $k>K / 2$.

Proposition 5 allows us to replace Assumptions 1-3 with the representation of $Q(\cdot \mid C)$ as a mixture of uniform autoregressions. Before we present a proof of Proposition 4 that exploits this result, we first develop three auxiliary results.

Definition 4. A function $f:[\hat{C}, \check{C}] \rightarrow \mathbb{R}$ is $\tilde{C}$-separable for some $\tilde{C} \in[\hat{C}, \check{C}]$, if (i) $f(C) \geq f(\tilde{C})$ for all $C>\tilde{C}$ and (ii) $f(C) \leq f(\tilde{C})$ for all $C<\tilde{C}$.

Lemma 1. Let $f:[\hat{C}, \check{C}] \rightarrow \mathbb{R}$ be integrable with respect to a uniform measure over its domain, $\tilde{C}$-separable, and nondecreasing on $[\hat{C}, \tilde{C}]$, for some $\tilde{C} \in[\hat{C}, \check{C}]$. Given a conditional probability distribution $Q(\cdot \mid C)$ for $C^{\prime}$ with nondecreasing expectation $\mu(C)$ that satisfies either

(i). $Q(\cdot \mid C)$ is degenerate at $\mu(C) \leq C$ for all $C \in[\hat{C}, \check{C}]$, or

(ii). $Q(\cdot \mid C)$ is uniform on $\left[\mu(C)-\frac{\sigma}{2}, \mu(C)+\frac{\sigma}{2}\right] \subseteq[\hat{C}, \check{C}]$ with $\sigma>0$ and $\mu(C)-\frac{\sigma}{2} \leq C$ for all $C \in[\hat{C}, \check{C}]$;

then $g(C) \equiv \int_{\hat{C}}^{\check{C}} f(c) d Q(c \mid C)$ is nondecreasing in $C$ on $[\hat{C}, \tilde{C}]$. 
Proof. In Case (i), the result follows immediately from $g(C)=f(\mu(C))$. Now consider Case (ii). First, note that $g(C)=\sigma^{-1} \int_{\mu(C)-\sigma / 2}^{\mu(C)+\sigma / 2} f(c) d c$. Because $f$ is nondecreasing on $[\hat{C}, \tilde{C}]$, it immediately follows that $g$ is nondecreasing on $\{C \in[\hat{C}, \tilde{C}] \mid \mu(C)+\sigma / 2 \leq \tilde{C}\}$. Next, for $C^{\dagger} \leq C \leq \tilde{C}$ such that $\mu\left(C^{\dagger}\right)+\sigma / 2 \geq \tilde{C}$, we have that

$$
\begin{aligned}
\sigma\left(g(C)-g\left(C^{\dagger}\right)\right) & =\int_{\mu\left(C^{\dagger}\right)+\sigma / 2}^{\mu(C)+\sigma / 2} f(c) d c-\int_{\mu\left(C^{\dagger}\right)-\sigma / 2}^{\mu(C)-\sigma / 2} f(c) d c \\
& \geq \int_{\mu\left(C^{\dagger}\right)+\sigma / 2}^{\mu(C)+\sigma / 2} f(\tilde{C}) d c-\int_{\mu\left(C^{\dagger}\right)-\sigma / 2}^{\mu(C)-\sigma / 2} f(c) d c \\
& \geq \int_{\mu\left(C^{\dagger}\right)+\sigma / 2}^{\mu(C)+\sigma / 2} f(\tilde{C}) d c-\int_{\mu\left(C^{\dagger}\right)-\sigma / 2}^{\mu(C)-\sigma / 2} f(\tilde{C}) d c \\
& =0 .
\end{aligned}
$$

Here, the first inequality uses that the first integral is over an interval to the right of $\tilde{C}$ and that $f$ is $\tilde{C}$ separable. The second inequality uses that the second integral is over an interval to the left of $\tilde{C}$ and that $f$ is nondecreasing on $[\hat{C}, \tilde{C}]$. Taken together, this implies that $g$ is nondecreasing on $[\hat{C}, \tilde{C}]$.

Lemma 2. Let $f:[\hat{C}, \check{C}] \rightarrow \mathbb{R}$ and $\tilde{C}$ satisfy the conditions of Lemma 1. If $Q^{K}(\cdot \mid C)=\sum_{k=1}^{K} p_{k}^{K} Q_{k}^{K}(\cdot \mid C)$ for some positive $p_{1}^{K}, \ldots, p_{K}^{K}$ and $Q_{1}^{K}(\cdot \mid C), \ldots, Q_{K}^{K}(\cdot \mid C)$ that each individually satisfy the conditions of Lemma 1, then $g^{K}(C) \equiv \int_{\hat{C}}^{\check{C}} f(c) d Q^{K}(c \mid C)$ is nondecreasing in $C$ on $[\hat{C}, \tilde{C}]$.

Proof. Lemma 1 implies that $g_{k}(C) \equiv \int_{\hat{C}}^{\check{C}} f(c) d Q_{k}(c \mid C)$ is nondecreasing on $[\hat{C}, \tilde{C}], k=1, \ldots, K$. In turn, because $g^{K}(C)=\sum_{k=1}^{K} p_{k} g_{k}(C)$, this implies that $g^{K}(C)$ is nondecreasing on $[\hat{C}, \tilde{C}]$.

Lemma 3. Let $f:[\hat{C}, \check{C}] \rightarrow \mathbb{R}$ be bounded, $\tilde{C}$-separable, and nondecreasing on $[\hat{C}, \tilde{C}]$, for some $\tilde{C} \in(\hat{C}, \check{C}]$. Let $Q^{1}, Q^{2}, \ldots$ be a sequence of mixture Markov transition functions satisfying the conditions of Lemma 2 such that $\sup \left|Q^{K}-Q\right| \rightarrow 0$ for some Markov transition distribution function $Q$ as $K \rightarrow \infty$. Then, $g(C) \equiv \int_{\hat{C}}^{\breve{C}} f(c) d Q(c \mid C)$ is nondecreasing in $C$ on $[\hat{C}, \tilde{C}]$.

Proof. Lemma 2 implies that the function $g^{K}$ corresponding to each $Q^{K} ; K=1,2, \ldots$; is nondecreasing on $[\hat{C}, \tilde{C}]$. By the bounded convergence theorem (Billingsley, 1995, Theorem 16.5), $g^{K} \rightarrow g$ as $K \rightarrow \infty$. Because the set of nondecreasing functions is closed, $g$ is nondecreasing on $[\hat{C}, \tilde{C}]$.

We are now prepared to present the proof of Proposition 4, using Proposition 5's mixture representation of $Q(\cdot \mid C)$.

Proof of Proposition 4. By Proposition 2, the unique equilibrium strategy is characterized by the survival sets $\mathcal{S}_{R^{\prime}} \equiv\left\{C \mid w_{R^{\prime}}(0, C)>0\right\}$ and the entry sets $\mathcal{E}_{R^{\prime}} \equiv\left\{C \mid w_{R^{\prime}}(0, C)>\varphi\left(R^{\prime}\right)\right\}$, with $w_{R^{\prime}}$ the unique fixed point of the Bellman operator $T_{R^{\prime}}$ defined by Equation (2); for $R^{\prime}=1, \ldots, N$. We need to show that there exist thresholds $\underline{C}_{R^{\prime}}$ and $\bar{C}_{R^{\prime}}$ such that $\mathcal{S}_{R^{\prime}}=\left\{C \mid C>\underline{C}_{R^{\prime}}\right\}$ and $\mathcal{E}_{R^{\prime}}=\left\{C \mid C>\bar{C}_{R^{\prime}}\right\} ; R^{\prime}=1, \ldots, \check{N}$.

Characterization of $\mathcal{S}_{\check{N}}$ and $\mathcal{E}_{\check{N}}$ Assumptions 1, 2, and 4; and the bounded convergence theorem (Billingsley, 1995, Theorem 16.5); imply that the operator $T_{\check{N}}$ maps the complete subspace of functions in $\mathcal{W}$ that are nondecreasing and continuous from the left in $C$ into itself. Consequently, the value function $w_{\tilde{N}}(X, C)$ is nondecreasing and continuous from the left in $C$. It immediately follows that there exist thresholds $\underline{C}_{\tilde{N}}$ and $\bar{C}_{\check{N}}$ such that $\mathcal{S}_{\check{N}}=\left\{C \mid C>\underline{C}_{\check{N}}\right\}$ and $\mathcal{E}_{\check{N}}=\left\{C \mid C>\bar{C}_{\check{N}}\right\}$. Note that either of these thresholds might equal $\hat{C}^{-}$or $\breve{C}$, where $\hat{C}^{-}$is an arbitrary number strictly below $\hat{C}$ representing the rule to be active in all demand states (including $\hat{C}$ ). 
Characterization of $\mathcal{S}_{R^{\prime}}$ and $\mathcal{E}_{R^{\prime}}$ for $R^{\prime}=\check{N}-1, \ldots, 1$ This proceeds iteratively. Suppose that; for $\tilde{R}=R^{\prime}+1, \ldots, \tilde{N}$; there exist thresholds $\underline{C}_{\tilde{R}}$ and $\bar{C}_{\tilde{R}}$ such that $\mathcal{S}_{\tilde{R}}=\left\{C \mid C>\underline{C}_{\tilde{R}}\right\}$ and $\mathcal{E}_{\tilde{R}}=\left\{C \mid C>\bar{C}_{\tilde{R}}\right\}$. Moreover, suppose that $w_{R^{\prime}+1}(0, C)$ is nondecreasing in $C$ for all $C \leq \bar{C}_{R^{\prime}+1}$ and that $w_{R^{\prime}+1}(X, C)$ is continuous from the left in $C$. We will show that then there exist thresholds $\underline{C}_{R^{\prime}}$ and $\bar{C}_{R^{\prime}}$ such that $\mathcal{S}_{R^{\prime}}=\left\{C \mid C>\underline{C}_{R^{\prime}}\right\}, \mathcal{E}_{R^{\prime}}=\left\{C \mid C>\bar{C}_{R^{\prime}}\right\}, w_{R^{\prime}}(0, C)$ is nondecreasing in $C$ for all $C \leq \bar{C}_{R^{\prime}}$, and $w_{R^{\prime}}(X, C)$ is continuous from the left in $C$. There are two cases to consider.

(i). In the first, $\mathcal{E}_{R^{\prime}+1}=\emptyset$, so that a firm entering with rank $R^{\prime}$ expects no further entry to occur during its lifetime. This case is identical to the case where $R^{\prime}=\check{N}$, so there exist thresholds $\underline{C}_{R^{\prime}}$ and $\bar{C}_{R^{\prime}}$ such that $\mathcal{S}_{R^{\prime}}=\left\{C \mid C>\underline{C}_{R^{\prime}}\right\}$ and $\mathcal{E}_{R^{\prime}}=\left\{C \mid C>\bar{C}_{R^{\prime}}\right\}, w_{R^{\prime}}(0, C)$ is nondecreasing in $C$, and $w_{R^{\prime}}(X, C)$ is continuous from the left in $C$.

(ii). In the second case, $\mathcal{E}_{R^{\prime}+1} \neq \emptyset$. The supposition that all firms with ranks $R^{\prime}+1$ and up use threshold rules ensures that $N_{R^{\prime}}^{\prime}(X, C)$ is continuous from the left in $C$. With Assumption 4 and the bounded convergence theorem, this implies that the operator $T_{R^{\prime}}$ maps the complete subspace of functions in $\mathcal{W}$ that are continuous from the left in $C$ into itself. Consequently, $w_{R^{\prime}}(X, C)$ is continuous from the left in $C$.

To prove the remaining results, we consider two subcases.

(a) In the first, $w_{R^{\prime}}(0, C)>\varphi\left(R^{\prime}\right)$ for all $C$, so we can set $\underline{C}_{R^{\prime}}=\bar{C}_{R^{\prime}}=\hat{C}^{-}$, for any $\hat{C}^{-}<\hat{C}$. Trivially, $w_{R^{\prime}}(0, C)$ is nondecreasing in $C$ for all $C \leq \bar{C}_{R^{\prime}}$.

(b) In the second subcase, $w_{R^{\prime}}(0, C) \leq \varphi\left(R^{\prime}\right)$ for some $C$. The argument for this subcase requires the construction of an auxiliary sequence of value functions by iterating on the Bellman operator $T_{R^{\prime}}$. To this end, initialize $w_{R^{\prime}}^{1}(X, C) \equiv w_{R^{\prime}+1}(\max \{X-1,0\}, C)$. Then; for $i=2,3, \ldots$; set $w_{R^{\prime}}^{i} \equiv T_{R^{\prime}}\left(w_{R^{\prime}}^{i-1}\right)$. This sequence converges to the unique fixed point $w_{R^{\prime}}$ of $T_{R^{\prime}}$. We first show that, for all $i \geq 1, w_{R^{\prime}}^{i+1} \geq w_{R^{\prime}}^{i}$ and $w^{i}(X, C)$ is continuous from the left in $C$.

- From the proof of Proposition 1, recall the definition of $\mathcal{W}_{R^{\prime}}$, the space of all functions $w(X, C) \in \mathcal{W}$ that are weakly decreasing in $X$ for all $C$ and satisfy $w(X+1, C) \geq w_{R^{\prime}+1}(X, C)$, $X=0, \ldots, \Sigma N-2$. In that proof, we showed that $T_{R^{\prime}}$ maps $\mathcal{W}_{R^{\prime}}$ into itself. Note that $w_{R^{\prime}}^{1}(X, C)$ is weakly decreasing in $X$ and $w_{R^{\prime}}^{1}(X+1, C)=w_{R^{\prime}+1}(X, C)$, so that $w_{R^{\prime}}^{1} \in \mathcal{W}_{R^{\prime}}$ and $w_{R^{\prime}}^{2}=T_{R^{\prime}}\left(w_{R^{\prime}}^{1}\right) \in \mathcal{W}_{R^{\prime}}$. Consequently,

- $w_{R^{\prime}}^{2}(X, C) \geq w_{R^{\prime}+1}(X-1, C)=w_{R^{\prime}}^{1}(X, C)$ for all $X \geq 1$, and

- $w_{R^{\prime}}^{2}(0, C) \geq w_{R^{\prime}}^{2}(1, C) \geq w_{R^{\prime}+1}(0, C)=w_{R^{\prime}}^{1}(0, C)$.

That is, $w_{R^{\prime}}^{2}=T_{R^{\prime}}\left(w_{R^{\prime}}^{1}\right) \geq w_{R^{\prime}}^{1}$. Since $T_{R^{\prime}}$ is monotonic, induction yields $w_{R^{\prime}}^{i+1} \geq w_{R^{\prime}}^{i}$ for all $i \geq 1$.

- By supposition, $w_{R^{\prime}}^{1}(X, C)$ is continuous from the left in $C$. Because $T_{R^{\prime}}$ maps the space of functions that are continuous from the left in $C$ into itself, this property carries over to $w_{R^{\prime}}^{i}(X, C)$ for all $i$.

We next show with induction that $w_{R^{\prime}}^{i}(0, C)$ and $\bar{C}_{R^{\prime}}^{i} \equiv \inf \left\{C \mid w_{R^{\prime}}^{i}(0, C)>\varphi\left(R^{\prime}\right)\right\} \leq \bar{C}_{R^{\prime}}^{i-1}$ together satisfy the conditions for $f(C)$ and $\tilde{C}$ in Lemma 3, and that $w_{R^{\prime}}^{i}(0, C)>\varphi\left(R^{\prime}\right)$ for all $C \in\left(\bar{C}_{R^{\prime}}^{i}, \check{C}\right]$. By supposition, $w_{R^{\prime}}^{1}(0, C)=w_{R^{\prime}+1}(0, C)$ and $\bar{C}_{R^{\prime}+1}$ together satisfy the conditions for $f(C)$ and $\tilde{C}$ in Lemma 3 , and $w_{R^{\prime}}^{1}(0, C)>\varphi\left(R^{\prime}+1\right)$ for $C \in\left(\bar{C}_{R^{\prime}+1}, \check{C}\right]$. Since $w_{R^{\prime}+1}(0, C)$ is continuous from the left, $w_{R^{\prime}}^{1}\left(0, \bar{C}_{R^{\prime}+1}\right) \leq \varphi\left(R^{\prime}+1\right)$. Because $\varphi\left(R^{\prime}+1\right) \geq \varphi\left(R^{\prime}\right)$, and $w_{R^{\prime}}^{1}(0, C)$ is nondecreasing and continuous from the left for $C \in\left[\hat{C}, \bar{C}_{R^{\prime}+1}\right]$, the conditions for Lemma 3 continue to hold good after replacing $\bar{C}_{R^{\prime}+1}$ with $\bar{C}_{R^{\prime}}^{1} \leq \bar{C}_{R^{\prime}+1}$. Moreover, $w_{R^{\prime}}^{1}(0, C)>$ $\varphi\left(R^{\prime}+1\right) \geq \varphi\left(R^{\prime}\right)$ for $C \in\left(\bar{C}_{R^{\prime}+1}, \check{C}\right]$. To show that $w_{R^{\prime}}^{1}(0, C)>\varphi\left(R^{\prime}\right)$ for $C \in\left(\bar{C}_{R^{\prime}}^{1}, \bar{C}_{R^{\prime}+1}\right]$ as well, note that $w_{R^{\prime}}^{1}(0, C)$ is nondecreasing on this interval and that, for any $\delta>0$, there exists a point $C^{\dagger} \in\left(\bar{C}_{R^{\prime}}^{1}, \bar{C}_{R^{\prime}}^{1}+\delta\right)$ such that $w_{R^{\prime}}^{1}\left(0, C^{\dagger}\right)>\varphi\left(R^{\prime}\right)$. Consequently, $w_{R^{\prime}}^{1}(0, C)>\varphi\left(R^{\prime}\right)$ for all $C \in\left(\bar{C}_{R^{\prime}}^{1}, \check{C}\right]$.

Continuing, suppose that $w_{R^{\prime}}^{i-1}(0, C)$ and $\bar{C}_{R^{\prime}}^{i-1}$ satisfy Lemma 3's requirements for $f(C)$ and $\tilde{C}$, and that $w_{R^{\prime}}^{i-1}(0, C)>\varphi\left(R^{\prime}\right)$ for all $C \in\left(\bar{C}_{R^{\prime}}^{i-1}, \check{C}\right]$. Lemma 3 then implies that $\mathbb{E}\left[w_{R^{\prime}}^{i-1}\left(0, C^{\prime}\right) \mid C\right]$ 
is nondecreasing in $C$ on $\left[\hat{C}, \bar{C}_{R^{\prime}}^{i-1}\right]$. This and inspection of Equation (2) determine that $w_{R^{\prime}}^{i}(0, C)$ is nondecreasing in $C$ on the same interval. Because $w_{R^{\prime}}^{i}(0, C) \geq w_{R^{\prime}}^{i-1}(0, C)$, we have that $\bar{C}_{R^{\prime}}^{i} \leq \bar{C}_{R^{\prime}}^{i-1}$ and that $w_{R^{\prime}}^{i}(0, C)>\varphi\left(R^{\prime}\right)$ for $C \in\left(\bar{C}_{R^{\prime}}^{i-1}, \check{C}\right]$. To show that $w_{R^{\prime}}^{i}(0, C)>\varphi\left(R^{\prime}\right)$ for $C \in\left(\bar{C}_{R^{\prime}}^{i}, \bar{C}_{R^{\prime}}^{i-1}\right]$ as well, note that $w_{R^{\prime}}^{i}(0, C)$ is nondecreasing on this interval and that, for any $\delta>0$, there exists a point $C^{\dagger} \in\left(\bar{C}_{R^{\prime}}^{i}, \bar{C}_{R^{\prime}}^{i}+\delta\right)$ such that $w_{R^{\prime}}^{i}\left(0, C^{\dagger}\right)>\varphi\left(R^{\prime}\right)$. Consequently, the function must exceed $\varphi\left(R^{\prime}\right)$ over the entire interval. Thus, $w_{R^{\prime}}^{i}(0, C)$ and $\bar{C}_{R^{\prime}}^{i}$ satisfy Lemma 3's requirements of $f(C)$ and $\tilde{C}$ and $w_{R^{\prime}}^{i}(0, C)>\varphi\left(R^{\prime}\right)$ for all $C \in\left(\bar{C}_{R^{\prime}}^{i}, \check{C}\right]$. Here, we use that $w_{R^{\prime}}^{i}(0, C)$ is continuous from the left to ensure that $w_{R^{\prime}}^{i}\left(0, \bar{C}_{R^{\prime}}^{i}\right) \leq \varphi\left(R^{\prime}\right)$.

Define $\bar{C}_{R^{\prime}}=\lim _{i \rightarrow \infty} \bar{C}_{R^{\prime}}^{i}$. We wish to show that

- $w_{R^{\prime}}(0, C) \leq \varphi\left(R^{\prime}\right)$ and is nondecreasing in $C$ for all $C \in\left[\hat{C}, \bar{C}_{R^{\prime}}\right]$ and

- $w_{R^{\prime}}(0, C)>\varphi\left(R^{\prime}\right)$ for all $C \in\left(\bar{C}_{R^{\prime}}, \check{C}\right]$.

The first assertion holds trivially if $\bar{C}_{R^{\prime}}=\hat{C}$, so focus on the case where $\bar{C}_{R^{\prime}}>\hat{C}$. Note that $w_{R^{\prime}}^{i}(0, C)$ is nondecreasing in $C$ and weakly less than $\varphi\left(R^{\prime}\right)$ on $\left[\hat{C}, \bar{C}_{R^{\prime}}^{i}\right]$ for all $i$. Because $\bar{C}_{R^{\prime}} \leq \bar{C}_{R^{\prime}}^{i}$, for all $C^{\dagger} \leq C \leq \bar{C}_{R^{\prime}}$,

$$
w_{R^{\prime}}(0, C)=\lim _{i \rightarrow \infty} w_{R^{\prime}}^{i}(0, C) \leq \varphi\left(R^{\prime}\right)
$$

and

$$
w_{R^{\prime}}\left(0, C^{\dagger}\right)=\lim _{i \rightarrow \infty} w_{R^{\prime}}^{i}\left(0, C^{\dagger}\right) \leq \lim _{i \rightarrow \infty} w_{R^{\prime}}^{i}(0, C)=w_{R^{\prime}}(0, C) .
$$

For the second assertion's demonstration, recall that $w_{R^{\prime}}^{i}(0, C)>\varphi\left(R^{\prime}\right)$ for all $C \in\left(\bar{C}_{R^{\prime}}, \check{C}\right]$. The definition of $\bar{C}_{R^{\prime}}$ implies that we can find an $i$ for any given $C>\bar{C}_{R^{\prime}}$ such that $w_{R^{\prime}}^{i}(0, C)>\varphi\left(R^{\prime}\right)$. Since the sequence $\left\{w_{R^{\prime}}^{i}(0, C)\right\}$ is nondecreasing, its limit $w_{R^{\prime}}(0, C)$ must also exceed $\varphi\left(R^{\prime}\right)$.

With this established, clearly $\mathcal{E}_{R^{\prime}}=\left\{C \mid C>\bar{C}_{R^{\prime}}\right\}$. Define

$$
\underline{C}_{R^{\prime}} \equiv \sup \left\{C \mid w_{R^{\prime}}(0, C) \leq 0\right\}
$$

if $\left\{C \mid w_{R^{\prime}}(0, C) \leq 0\right\} \neq \emptyset$, and $\underline{C}_{R^{\prime}} \equiv \hat{C}^{-}$otherwise. By construction, $\underline{C}_{R^{\prime}} \leq \bar{C}_{R^{\prime}}$. Because $w_{R^{\prime}}(0, C)$ is nondecreasing and continuous from the left for $C \in\left[\hat{C}, \bar{C}_{R^{\prime}}\right]$, we can write $\mathcal{S}_{R^{\prime}}=$ $\left\{C \mid C>\underline{C}_{R^{\prime}}\right\}$.

\section{References}

Abbring, J. H. and J. R. Campbell (2006). A firm's first year. Federal Reserve Bank of Chicago Working Paper 2003-11. 13

Abbring, J. H. and J. R. Campbell (2007). Duopoly dynamics with a barrier to entry. Federal Reserve Bank of Chicago Working Paper 2006-29. 1, 2, 3, 9

Amir, R. and V. E. Lambson (2003). Entry, exit, and imperfect information in the long run. Journal of Economic Theory 110, 191-203. 6

Berry, S. (1992). Estimation of a model of entry in the airline industry. Econometrica 60(4), 889-917. 1, 13

Billingsley, P. (1995). Probability and Measure (Third ed.). New York: Wiley. 22, 24

Bresnahan, T. F. and P. C. Reiss (1990). Entry in monopoly markets. Review of Economic Studies 57(4), 531-553. 1, 2, 3, 13, 15

Bresnahan, T. F. and P. C. Reiss (1991a). Empirical models of discrete games. Journal of Econometrics 48(12), 57-81. 1 
Bresnahan, T. F. and P. C. Reiss (1991b, October). Entry and competition in concentrated markets. Journal of Political Economy 99(5), 977-1009. 13, 15, 17, 18

Bresnahan, T. F. and P. C. Reiss (1993). Measuring the importance of sunk costs. Annales d'Economie et de Statistique 31, 181-217. 1

Cabral, L. M. (1993). Experience advantages and entry dynamics. Journal of Economic Theory 59, 403-416. 2

Dixit, A. K. (1980, March). The role of investment in entry-deterrence. Economic Journal 90(357), 95-106. 2

Dixit, A. K. and R. S. Pindyck (1994). Investment under Uncertainty. Princeton, NJ: Princeton University Press. 2, 13

Doraszelski, U. and M. Satterthwaite (2007). Computable Markov-perfect industry dynamics: Existence, purification, and multiplicity. Unpublished Manuscript, Harvard University. 5

Dunne, T., M. J. Roberts, and L. Samuelson (1988). Patterns of firm entry and exit in U.S. manufacturing industries. Rand Journal of Economics 19, 495-515. 5

Ericson, R. and A. Pakes (1995). Markov-perfect industry dynamics: A framework for empirical work. Review of Economic Studies 62, 53-82. 2

Feller, W. (1971). An Introduction to Probability Theory and Its Applications (2nd ed.), Volume II. New York, NY: Wiley. 22, 23

Fudenberg, D. and J. Tirole (1991). Game Theory. Cambridge, MA: MIT Press. 20

Hopenhayn, H. A. (1992). Entry, exit, and firm dynamics in long run equilibrium. Econometrica 60(5), 1127-1150. 2, 9

Jarmin, R. S., S. D. Klimek, and J. Miranda (2003, October). Firm entry and exit in the U.S. Retail sector: 1977-1997. Center for Economic Studies, U.S. Bureau of the Census, CES-WP-04-17. 5

Jovanovic, B. (1982). Selection and the evolution of industry. Econometrica 50(3), 649-670. 2

Maskin, E. and J. Tirole (1988). A theory of dynamic oligopoly, I: Overview and quantity competition with large fixed costs. Econometrica 56(3), 549-569. 2

McAfee, R. P., H. M. Mialon, and M. A. Williams (2004). What is a barrier to entry? American Economic Review 94(2), 461-465. 13

Pakes, A. and P. McGuire (1994). Computing Markov-perfect Nash equilibria: Numerical implications of a dynamic differentiated product model. The RAND Journal of Economics 25(4), 555-589. 2

Sutton, J. (1991). Sunk Costs and Market Structure. Cambridge, MA: MIT Press. 13 